\title{
Secondary craters and ejecta across the solar system: Populations and effects on impact-crater-based chronologies
}

\author{
E. B. BIERHAUS (iD ${ }^{1, *}$, A. S. McEwen ${ }^{2}$, S. J. ROBBINS (iD ${ }^{3}$, K. N. SINGER ${ }^{3}$, L. DONES $^{3}$, \\ M. R. KIRCHOFF (iD) ${ }^{3}$, and J.-P. WILLIAMS (iD ${ }^{4}$ \\ ${ }^{1}$ Lockheed Martin Space, Denver, Colorado, USA \\ ${ }^{2}$ University of Arizona, Tucson, Arizona, USA \\ ${ }^{3}$ Southwest Research Institute, Boulder, Colorado, USA \\ ${ }^{4}$ University of California, Los Angeles, California, USA \\ *Corresponding author. E-mail: edward.b.bierhaus@1mco.com
}

(Received 02 January 2017; revision accepted 15 January 2018)

\begin{abstract}
We review the secondary-crater research over the past decade, and provide new analyses and simulations that are the first to model an accumulation of a combined primary-plus-secondary crater population as discrete cratering events. We develop the secondary populations by using scaling laws to generate ejecta fragments, integrating the trajectories of individual ejecta fragments, noting the location and velocity at impact, and using scaling laws to estimate secondary-crater diameters given the impact conditions. We also explore the relationship between the impactor size-frequency distribution (SFD) and the resulting secondary-crater SFD. Our results from these analyses indicate that the "secondary effect" varies from surface to surface and that no single conclusion applies across the solar system nor at any given moment in time-rather, there is a spectrum of outcomes both spatially and temporally, dependent upon target parameters and the impacting population. Surface gravity and escape speed define the spatial distribution of secondaries. A shallow-sloped impactor SFD will cause proportionally more secondaries than a steeper-sloped SFD. Accounting for the driving factors that define the magnitude and spatial distribution of secondaries is essential to determine the relative population of secondary craters, and their effect on derived surface ages.
\end{abstract}

\section{INTRODUCTION AND HISTORIC CONTEXT}

\section{Impact Crater Populations, and the Application of Craters as Chronological Markers}

Impact craters are the most abundant landforms in the solar system. Impact craters are observed on almost every solid surface from Mercury to Pluto and Charon, and across target bodies visited by spacecraft that range from the roughly $0.5 \mathrm{~km}$ asteroid Itokawa to moons and planets that are thousands of $\mathrm{km}$ in diameter. The single impact-crater-less (as far as we know) exception is Jupiter's moon Io, which we discuss further in later sections. Craters range in size from microscopic pits to enormous basins several thousand $\mathrm{km}$ in diameter. Because of their ubiquity in space and in time, impact craters are central to understanding the evolution of planetary surfaces.

Impact craters form from projectiles striking a surface with sufficient energy and momentum to excavate surface material and create a cavity. There are several sources of projectiles, each of which form a corresponding crater population: primary craters, secondary craters, and sesquinary craters.

Primary impactors are comets and asteroids that strike a surface to make primary craters. (Primary craters include impacts from human-made objects; for instance, many past spacecraft deliberately ended their missions by impacting the Moon, see e.g., Wagner et al. 2017.) During the formation of a primary crater, material is ejected from the target; craters formed by material ejected from a primary crater with sufficient 
reimpact speed to also form craters are known as secondary craters, also called simply "secondaries." (Secondary-crater formation requires that the escape speed of the body be greater than the minimum speed necessary to make a secondary crater.) In some cases, the speed of an ejected fragment exceeds the escape velocity of the target; if this fragment subsequently strikes its source, or some other object, after following an independent trajectory through space, the resulting crater is called a sesquinary crater (Zahnle et al. 2008). These are called sesquinary, or "one-and-a-half," craters because the sesquinary projectile comes from outside the target's Hill Sphere like a primary projectile, but the source of the impactor is the target itself like a secondary projectile.

Secondary craters are hardly a secondary consideration when evaluating the evolution of a planetary surface: the physics of the processes that eject fragments, the lower energy impact that makes secondaries, and their spatial and size-frequency distributions (SFDs) crosscut all aspects of crater studies. A single primary crater can broadcast up to $\sim 10^{6}$ or more secondaries (Bierhaus et al. 2001; McEwen et al. 2005; Dundas and McEwen 2007; Preblich et al. 2007) - depending on the size of the primary crater - in a geologic instant. These secondaries are distributed at least locally, and perhaps globally (Shoemaker et al. 1994). Crater ejecta in general, and secondary cratering in particular, are in effect a transmission of the primary impact energy to a much broader region than the primary impact site itself.

Crater populations, both primary and secondary, are often approximated as power laws over certain diameter ranges. Though a power law is a poor description over the full diameter range of the populations, power laws are usually reasonable approximations over specific diameter segments. In the differential power law formulation, craters have the size-frequency relationship $\frac{\mathrm{dN}}{\mathrm{dD}}=k D^{-b}$, where $\mathrm{d} N$ is the number of craters in the diameter range $\mathrm{d} D$, with representative diameter $D$, and $k$ and $b$ are constants derived by a fit to the data. The cumulative distribution is the integral of the differential distribution, or $N$ $\left(D>D^{\prime}\right)=c D^{-\alpha}$, where $N$ is the number of craters with diameter $D$ and larger, with $c=k /(1-b)$ and $\alpha=b-1$. The parameters $b$ and $\alpha$ are the power law indices for the differential and cumulative versions of the power laws, respectively. When plotted on a log-log plot, a power law forms a straight line with slope equivalent to its power law index. This relationship is the source of the now widespread use of the word "slope" used to describe a crater SFD; we continue that terminology here. A "steep" slope has a relatively large value of $\alpha$ or $b$.
In this paper we plot crater populations using the relative format, or "R-plot" (Crater Analysis Technical Working Group 1979). The relative format is the differential data normalized to a -3 differential slope (i.e., $b=3$ ), thus a -3 differential slope plots as a horizontal line in an R-plot (while a -2 differential slope is +1 slope in an $\mathrm{R}$-plot, and -4 differential slope is -1 slope in an R-plot). The R-plot is a useful tool to interpret crater SFDs: the vertical position of a data point corresponds to the spatial density of craters at that diameter bin (higher $R$-values mean higher spatial density); deviations from a single power law parameter are easily visible if they exist; and as a practical matter, it is a more compact means to display the data.

Researchers use craters to understand the physical characteristics of the target surface (by evaluating crater morphology), as well as derive estimates of relative and absolute surface ages. The principle behind impactcrater-based chronology is simple: the longer a surface is exposed to an impacting population, the more impacts it experiences. Thus, an older surface will have more craters than a younger surface, normalized by area. Comparing crater spatial densities between terrains gives relative ages; in the event the flux rate of impacts is known, then one can use the observed crater distribution to derive an absolute surface age. This simple picture becomes more complicated in the face of several factors, such as whether or not the stochastic addition of spatially random secondary craters to the observed crater population significantly changes a derived surface age (e.g., Williams et al., 2018). In addition to analysis of secondary-crater populations, data from long-lived orbiting spacecraft around Mars (Daubar et al. 2013, 2014) and the Moon (Speyerer et al. 2016) provide an unprecedented opportunity to evaluate both the Hartmann production function (HPF) and the Neukum production function (NPF) against the current impact rate (Hartmann and Daubar 2017).

\section{The "Secondary Effect" Debate}

The recognition of secondary craters as a fundamental component of crater populations began in the 1960s with Eugene Shoemaker's observations of lunar craters from Ranger images (Shoemaker 1965). $\mathrm{He}$ proposed that secondaries comprise a significant fraction of the small $(<1 \mathrm{~km})$ craters on the Moon, and wrote "With decreasing crater size below $200 \mathrm{~m}$, the predicted number of secondary craters rapidly becomes much greater than the number of primaries expected on the basis of extrapolation." Around this same time, Roberts (1964) examined ejecta fields surrounding ground-explosion craters on Earth, including the $100 \mathrm{kt}$ 
Sedan explosion (an underground nuclear detonation), and observed "Small secondary craters more than $5000 \mathrm{ft}$ from Sedan ground zero were noted during a search. ... Inside $5000 \mathrm{ft}$, these small craters, ranging in diameter from 1.5 to $6 \mathrm{ft}$, were too numerous to note on the map. In all probability, the total number of all secondary craters surrounding Sedan may be in the tens of thousands."

However, there was not universal agreement on the effect that secondaries have on a surface's overall crater SFD, and the implications for deriving a surface age, and the debate regarding the effect of secondary contamination of crater measurements and surface ages continued through the 1970 s, and indeed continues to the present day. Some workers concluded that secondaries dominated for diameters $<\sim 1 \mathrm{~km}$ on the Moon and Mars, and significant numbers of small circular craters could not be distinguished as primaries or secondaries based on morphology (Soderblom et al. 1974; Wilhelms et al. 1978; Carr 2006). Others argued that small circular craters are chiefly primaries, and that secondaries are easily identified and removed from crater counts (e.g., Neukum and Ivanov 1994; Neukum et al. 2001), or that obvious secondaries can be removed, and the remaining "background" secondaries accumulate in tandem with small primaries and preserve the validity of a crater-based chronology (Hartmann 2005 , 2007). The magnitude and variability of the "secondary effect" is central to understanding the ages derived from the two most common crater production functions used by the community, the HPF (see Hartmann [2005] for a recent description of the HPF as formulated for Mars) and the NPF (see Ivanov 2001).

The views of Neukum and Ivanov (1994) and others that secondaries are unimportant to chronology are based largely on the work of Neukum et al. (1975) and continue in more recent work (e.g., Werner et al. 2009). Neukum et al. (1975) tested the idea that shallow circular secondaries may contaminate primary crater populations by identifying craters in circular rings around Bessel crater (16 km diameter) in southern Mare Serenitatis (on Earth's Moon), concluding that (1) secondaries account for $\leq 30 \%$ of craters near $1 \mathrm{~km}$ diameter in the immediate vicinity of the primary, and (2) the secondary population rapidly falls to almost zero at a distance of 7 crater radii. However, the very largest secondary is expected to be $\sim 5 \%$ as large as the primary (Melosh 1989; Singer et al. 2015), so the $16 \mathrm{~km}$ diameter Bessel would not be expected to produce many, if any, secondary craters $>\sim 1 \mathrm{~km}$. (Sometimes the very largest secondaries can be as big as $10 \%$ the diameter of their parent primary [Robbins and Hynek 2011b], but there are never more than a few of these very largest secondaries.) Only primary craters larger than $\sim 100 \mathrm{~km}$ are expected to produce large numbers of secondaries $\geq 1 \mathrm{~km}$ on any solar system body. Nevertheless, Neukum et al. (1975) stated: "It is clear that the contribution of secondary craters can be neglected in the $\mathrm{km}$-size range for all areas which do not lie in the immediate vicinity of large primary craters." This result has been cited many times in subsequent papers (Neukum and Ivanov 1994; Neukum et al. 2001; Hartmann 2005) as justification for expecting secondaries to be $<10 \%$ of total crater numbers. Similar examples are in more recent work, such as Werner et al. (2009) who wrote, "the percentage of hypothetically globally unrecognized secondary craters is usually $<5 \%$ for any measurement and has a minor effect on ages ... [and we] conclude that crater count measurement are 'contaminated' by secondary cratering by percentages smaller than the assumed statistical error." Likewise, Quantin et al. (2016) studied secondaries around $7 \mathrm{~km}$ diameter Gratteri crater on Mars, and concluded that secondaries are unimportant to dating Noachian $(>3.7 \mathrm{Ga})$ terrains, and further concluded that secondaries are globally unimportant to chronology for ages $>\sim 20 \mathrm{Myr}$. As we will show below, the fact that small secondaries from a recent small primary crater have little effect on dating Noachian terrains should not be surprising. Rather, large secondaries from even larger Noachian primary craters and basins are of concern to dating Noachian terrains.

In the 1980 s to 1990 s, many workers who had argued for or against the significance of secondary craters moved on to other areas of research, and the question of the secondary-crater effect on crater-based chronologies lay mostly dormant. Meanwhile, the observation of a steeper SFD (differential power law exponent $b \sim 4.1-4.7$ ) from craters $\leq 1 \mathrm{~km}$ on Gaspra, where secondaries must be extremely rare or absent due to low surface gravity, was considered evidence for a steep primary SFD for small craters (Neukum and Ivanov 1994; Chapman et al. 1996). Evidence for a steep SFD for primaries was not new-Shoemaker (1965) reached the same conclusion based on meteorite fall data combined with crater measurements-but it did not prove that secondaries were unimportant when applying a crater-based chronology. Nevertheless, Neukum and Ivanov (1994) concluded “... we are dealing with a primary crater distribution over this whole range $[D \leq 10 \mathrm{~km}]$." Other review papers of crater-based chronologies (Hartmann and Neukum 2001; Neukum et al. 2001; Ivanov et al. 2002) made no mention of secondary craters.

Other papers were published in the 1980s that advanced our understanding of crater ejecta and secondary cratering. Melosh (1984) explained how a high-energy impact event generates fast-moving ejecta 
that remain lightly shocked through spallation of nearsurface material. Though a focus on the work was to explain the presence and physical state of lunar and Martian meteorites on Earth, the ejection mechanisms described in the analysis also provide a basis for understanding the production and distribution of secondary craters created from spall fragments (one type of high-speed ejected material). Schultz and Gault (1985) explored the evolution in crater morphology as a group of impactors changed from tightly clustered (approximating a single impactor) to more widely dispersed. A key finding was that less tightly clustered impactors lead to noticeable differences in crater morphology - in particular, resulting in more shallow craters, a possible explanation for the generally more shallow secondaries (see Bierhaus and Schenk [2010] and references therein). Vickery (1987) measured the size of secondary craters as a function of distance from their parent primary to derive fragment sizes, and although her data set was limited relative to today's standards, she found strong trends in the decrease of ejected fragment diameter (inferred from secondarycrater diameter) as function of distance from the primary crater.

The study of secondary craters got a revival in the new millennium. At least two developments led to this revival

1. Several new planetary-spacecraft missions (see Table 1) provided high-quality image data of planetary surfaces across the solar system, expanding areal coverage and increasing spatial resolution. These images enable crater measurements over larger surface areas and to smaller diameters. The higher resolution images of Mars (McEwen et al. 2005) and Europa (Bierhaus et al. 2005) led to nearly coincident conclusions that secondaries should dominate the small craters.

2. The discoveries on Earth of meteorites from the Moon and Mars required crater ejecta to exceed escape velocity, and because of the inverse relationship between ejected mass and ejection velocity, much more mass (i.e., more fragments) falls back to make secondary craters. Head et al. (2002) estimated that an impact event that delivered a discovered meteorite to Earth must have ejected at least $10^{6}$ rocks larger than $3 \mathrm{~cm}$ in diameter at greater than Mars escape velocity $\left(5 \mathrm{~km} \mathrm{~s}^{-1}\right)$. This work demonstrated that the production of fastmoving ejecta is a natural consequence of primary cratering, resulting in widespread ejecta.

Today, secondary craters are widely recognized as contributors to the total crater population, though there is no consensus on their effect on crater-based chronologies, and little work exists to describe how secondary crater populations may differ between planetary objects. In this work we have two goals. First, we summarize the current state of knowledge of secondary craters, reviewing literature in roughly the past decade (since the previous review, McEwen and Bierhaus 2006). Second, we provide new results that (1) explore the evolution of overall primary-plus-secondary crater SFDs using the most up-to-date information on secondary populations, and (2) investigate how the overall crater SFD will vary as a function of both the primary impact flux and the resulting secondary-crater population.

In support of those two goals, the remainder of the paper is structured as follows. In the Recent Work section, we focus on the work relevant to secondary cratering in roughly the last decade. In the New Results section, we present new work that seeks to improve the connection between theoretical expectations and actual observations. In the Consequences for Crater SFDs and Age Determination section, we describe the consequences of secondary craters when interpreting impact crater measurements, concluding that secondaries affect crater populations and companion surface age estimates on many solar system bodies when using craters at and smaller than the $\mathrm{km}$ scale, though the magnitude of their effect varies. Finally, we tie these together in last section and distill the characteristics of secondaries that are true regardless of planetary body, and those characteristics that will be unique to each planetary body.

\section{RECENT WORK}

Many studies utilize the crater populations visible in the data returned by the missions listed in Table 1 . Though not all of them address or consider secondary craters as a central topic of investigation, many authors use these data, as well as analytical considerations, to understand secondary cratering as a process, as well as the effect of secondaries on impact-crater SFDs. McEwen and Bierhaus (2006) summarized the recent discoveries and important considerations of secondary cratering at the time, as well as providing new insights -in particular, McEwen and Bierhaus (2006) showed that secondaries must produce a steeper SFD than primaries (over the size range creating the secondaries) because of the size-velocity relation of ejected fragments (Vickery 1986). Due to the significant increase in data sets across the solar system since 2006, there have been several studies since that review that investigate the relative abundance of secondaries and their effects on impact crater SFDs. In the following subsections, we review work completed during the decade since McEwen and Bierhaus (2006). First we review the secondary-crater research in order from the inner to 
Table 1. A partial list of planetary missions since the mid-1990s with attention to those that provided new image data of planetary surfaces.

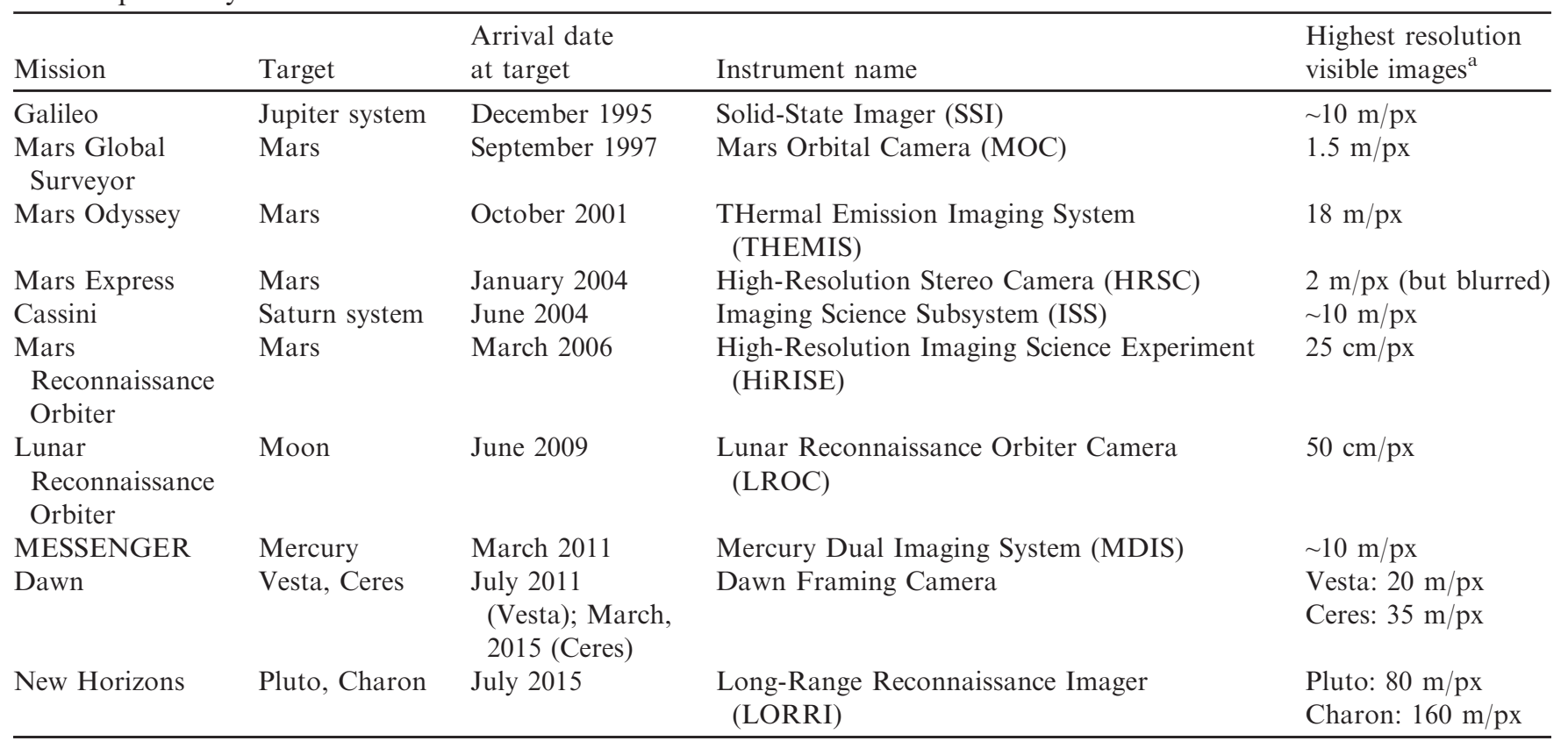

${ }^{\text {a }}$ The resolutions listed are representative of the highest resolutions achieved. In the case of missions that view multiple planetary objects (e.g., Galileo and Cassini), the range given represents the data available for multiple surfaces.

outer solar system, and then transition to studies that address broader-scale topics such as crater SFDs and implications for crater chronology.

\section{Data/Observations}

Fifty years after the first major publications on secondary craters, the debate over their abundance and effect has not disappeared. For those studies that use images to measure crater populations, there at least seems to be a consensus that secondary craters-in addition to obvious rays and clusters - are present in the "background" population up to tens of percent, even if there is not agreement over the maximum contribution they may add, or their effect on craterbased chronologies.

\section{Mercury Data}

Mariner 10 data of Mercury from the 1970s identified an SFD slope increase in the crater population of Mercury at $D<\approx 10 \mathrm{~km}$ (shallower for diameters $>10 \mathrm{~km}$, steeper for diameters $<10 \mathrm{~km}$ ), Strom (1977) attributed the $D<10 \mathrm{~km}$ crater density increase to secondary craters. From the first Science papers of MESSENGER's flybys of Mercury before entering orbit in 2011, Strom et al. (2008) again identified this steepening slope and attributed it to possible secondary craters.
While there are broad theoretical reasons to support the Strom et al. conclusions, and the shape of the SFD has been argued to support this interpretation (Strom et al. 2011), examination of the rich MESSENGER data set with particular focus on the secondary-crater population is still in the early stages. Xiao (2016) and Xiao et al. (2016) examined secondary craters on Mercury. Xiao (2016) found that self-secondaries, classical "ray" secondaries, and background secondaries are all abundant. A notable finding from the study is "Secondaries are widespread outside of the impact ray, and they are part of the distant secondary crater population, because they show a continuous extension from the main secondaries cluster within the rays. ... The density of secondaries gradually decreases perpendicularly outward from the ray over a distance of dozens of kilometers. Without the regional context that shows the impact ray nearby, such distant secondaries could be easily mistaken as primaries." This is similar to the conclusion reached by Preblich et al. (2007) when examining lunar rays. Xiao et al. (2016) focused on the self-secondary crater population of the young Hokusai crater; due to its small area and young age, they examined craters with $D \leq 0.1 \mathrm{~km}$. While they found a large and steep population of secondaries, and they concluded that craters of this size were unusable for age estimation, the measured craters are two orders of magnitude smaller than the $\approx 10 \mathrm{~km}$ SFD slope change. 
One ongoing study by one of the authors of this work (Robbins) includes classification of individual craters as primary or secondary over a large geologic mapping area. This study covers approximately $4 \%$ of Mercury's surface in support of a geologic mapping effort over the Rembrandt basin area, $50-15^{\circ} \mathrm{S}$ by 65 $115^{\circ} \mathrm{E}$ (Hynek et al. 2016). The area contains approximately 10,400 craters $D \geq 3 \mathrm{~km}$, though an additional $\approx 36,000$ craters were identified to ensure complete $D \geq 3 \mathrm{~km}$ statistics. The overall crater statistics of the region show the same kind of increased slope observed in previous work for $D<\approx 10 \mathrm{~km}$ (Strom et al. 2011). Robbins then separated only morphologically "obvious" secondary craters, using the common criteria of crater clustering, crater chains, herring-bone ejecta patterns, and highly elliptical craters with a major axis pointing to the primary.

Figure 1 is an R-plot of the results of this study. The $R$-value of the total crater population shows an increase for diameters $\leq 10 \mathrm{~km}$. The decrease in $R$-value at the smallest sizes is due to the finite resolution of the images, and not the behavior of the actual population; the completeness limit is between 3.5 and $4 \mathrm{~km}$ for the images used. The plot illustrates that the $R$-value increase at $\sim 10 \mathrm{~km}$ is due to the increasing population of secondaries for these sizes; the primary craters maintain a $-2.62 \pm 0.03$ differential slope, while the secondaries have a $-4.82 \pm 0.07$ differential slope. If these data are representative of the global crater populations, secondaries are more numerous than primaries below $5 \mathrm{~km}$.

The data in Fig. 1 are consistent with those presented in Xiao (2016), see his figs. 3 and 9, which compare the measurements from Ostrach et al. (2015) that have a slightly positive slope in R-format (corresponding to a slope more shallow than -3 differential) for craters $>10 \mathrm{~km}$, and a steeply sloped secondary population for crater diameters $<10 \mathrm{~km}$. In both studies, the secondaries in local areas reach saturation, a condition when the formation of a new crater on average destroys a pre-existing crater of similar size. (Saturation is in effect the maximum spatial density of craters a surface can sustain, which is also dependent on the SFD of the impacting population, see Chapman and McKinnon 1986.) When a surface is saturated, disentangling which craters are primary and secondary and evaluating the relative density is an extremely challenging task. But saturation also renders an accurate age estimate impossible (except that the surface is old enough to have reached saturation), so surfaces saturated with craters become divorced from the fundamental question of how much error background secondaries introduce into an age estimate. For unsaturated regions, the collective work done by

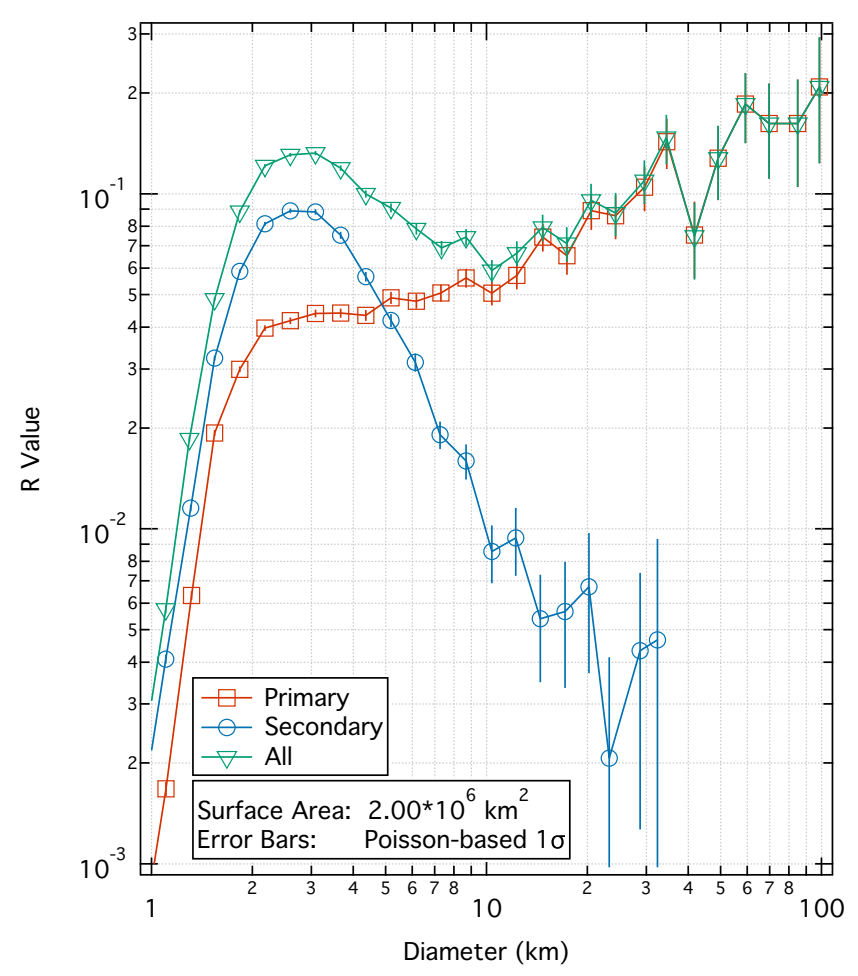

Fig. 1. The SFD for primary, secondary, and all craters from Mercury study area. These data indicate that the increase in the crater SFD seen at $\sim 10 \mathrm{~km}$ is due to the secondary crater population. The completeness limit for these data is $\sim 4 \mathrm{~km}$, the roll-off in crater density at smaller diameters is a resolution effect and not real. (Color figure can be viewed at wileyonlinelibrary.com.)

coauthor Robbins, who identified only obvious secondaries, and that of Xiao (2016), who found evidence for extensive background secondaries outside of crater rays, suggest that background secondaries are an important contributor to the observed crater population $<10 \mathrm{~km}$.

The interplay of the primary and secondary-crater SFDs of course depends on the SFD of the impacting population. Recent observations of near-Earth asteroids, or NEAs (Granvik et al. 2016), suggest that the low-albedo objects are more likely to disrupt as they achieve lower perihelion. There are several interesting connections that may exist between the crater SFD observations of Mercury and the nature of small-body populations within the inner solar system. The differential slope of primary craters on Mercury, illustrated by Fig. 1, is shallower than that of similar diameters on the Moon. In the event that low-albedo objects are rare or absent as impactors on Mercury, then comparing the primary-crater SFD on the Moon with that of Mercury may give some sense of the relative proportion of low- and high-albedo objects within the impacting population. More to the matter at 
hand, i.e., the role of secondaries within impact-crater SFDs, our simulations (see the New Results section) indicate that secondaries are more consequential to the small-crater population, and introduce greater error to impact-crater based chronologies, as the primary crater SFD becomes more shallow. If the impactor SFD on Mercury is shallower than the impactor SFD on the Moon and Mars, then secondaries on Mercury are more problematic for a crater-based chronology than for the steeper impactor SFD for the Moon and Mars.

An additional consideration in the abundance of large $(>1 \mathrm{~km})$ secondaries on Mercury is the age of major plains relative to heavy bombardment (McEwen et al. 2017a). Most intercrater plains on Mercury date to the period of heavy bombardment (Strom et al. 2008). Many large $(>200 \mathrm{~km})$ impact craters and basins were still being produced, leading to the abundant large $(>1 \mathrm{~km})$ secondaries, and not much happened since then to erase these secondaries. A comparable population of large secondaries could have been produced on the Moon and Mars during this time period, but would appear to have been erased or are difficult to recognize today. The Moon contains two main terrains: highland and maria. The ancient highlands are saturated with craters smaller than $\sim 10 \mathrm{~km}$, with an SFD that matches saturation equilibrium (Hartmann and Gaskell 1997). Secondaries from the youngest lunar impact basins have been recognized and mapped (Wilhelms et al. 1978), revealing the "steep" SFD expected for secondaries. The lunar maria is less than $3.5 \mathrm{Gyr}$ old, and there are no younger primary craters on the Moon larger than Hausen (167 km diameter), i.e., there is an absence of primaries capable of producing abundant secondaries $>1 \mathrm{~km}$ over the maria. On the young volcanic plains of Mars, the largest primary young enough to contribute significant numbers of large secondaries is $\sim 100 \mathrm{~km}$ diameter (Strom et al. 2015). In addition, extensive erosion at the Noachian/Hesperian boundary $(\approx 3.6-$ 3.7 Ga; Irwin et al. 2013; Michael 2013) has erased craters smaller than tens of $\mathrm{km}$ diameter on Noachianaged terrains, including the basin secondaries. The only impact basins on Mars that postdate this period of erosion are Lyot and Lowell, which do exhibit a significant population of large secondary craters (Robbins and Hynek 2011a, 2011b, 2014).

\section{Lunar Data}

In recent years, the Lunar Reconnaissance Orbiter has provided a rich data set to revisit the crater measurements that began in the Apollo era, provided high-resolution data sets over much larger areas, and even made the first direct estimates of the current impact rate.

Several studies have concluded that secondary craters are unimportant for the $\sim \mathrm{km}$-scale lunar crater population. Kirchoff et al. (2013) examined the superposed craters on the floors of large impact craters near $100 \mathrm{~km}$ diameter; their measurements for the superposed craters are generally for diameters $>600 \mathrm{~m}$. When obvious secondaries were removed from the measurements, the resulting crater SFDs for most of the younger terrains examined in this study matched the Marchi et al. (2009) production function, which is derived from models and observations of NEOs - not craters-indicating little potential contribution by background secondaries in this diameter range. However, background secondaries are likely abundant at smaller diameters, as described in Shoemaker's original work. Both Ivanov (2006) and Williams et al. (2014b) used bolide measurements reported in Brown et al. (2002), in conjunction with crater scaling laws, to estimate the small primary-crater population on the Moon and Mars. Ivanov (2006) concluded that the data are most consistent with secondaries comprising well below $25-50 \%$ (or up to $75 \%$ using what he terms an unrealistic scaling) of the $<200 \mathrm{~m}$ craters. In contrast, the similar study in Williams et al. (2014b) concluded: "Since our Monte Carlo simulations demonstrate that the existing crater chronology systems can be applied to date young surfaces using small craters on the Moon and Mars, we conclude that the signal from secondary craters in the isochrons must be relatively small at these locations, as our Monte Carlo model only generates primary craters."

Other studies concluded that secondaries are more consequential to the small lunar population. Namiki and Honda (2003) re-examined the steepening of the lunar SFD $<3 \mathrm{~km}$ diameter, and they concluded that secondary craters were the likely cause. Hirata and Nakamura (2006) reanalyzed Tycho secondaries and concluded that there are many small secondary craters far from the primary crater and that "A dominant contribution of secondaries to the SFD of all lunar craters is thus strongly suggested." Dundas and McEwen (2007) concluded that Tycho produced almost $10^{6}$ secondaries larger than $63 \mathrm{~m}$ diameter, in spite of forming in the heavily fragmented highlands over which impact spallation is expected to be less important (Melosh 1984; Hartmann and Barlow 2006). Wells et al. (2010) showed that secondary craters that are not obvious secondaries in visible images could be distinguished by circular polarization ratio radar images, providing further evidence that unrecognizable secondaries are embedded in the sub-km crater population. Strom et al. (2015) reiterated the view that secondary craters can dominate the cratering record at small diameters.

The new data suggest that the SFDs of secondaries exhibit significant variability, even if there is a useful 
characterization that their SFDs are steep. The SFD slope for Copernicus secondaries mapped in Singer et al. (2014) displays a very steep cumulative slope of -6 to -4 depending on which portion of the size range was fit (they studied $0.2-6 \mathrm{~km}$ diameter secondaries). Their study focused on more obvious secondaries: those in chains or clusters with clear radial indicators such as $\mathrm{v}$-shaped ejecta pointing back to the parent crater. Kirchoff et al. (2013) found that small secondary clusters can have a wide range in crater SFD slope from very shallow to very steep. Krishna and Kumar (2016) performed an extensive, detailed study of the boulder and secondary-crater population around a $3.8 \mathrm{~km}$ primary crater. They found variation in the slope of the secondaries, ranging from $\sim-3$ cumulative to $\sim-4.5$ cumulative. The steep slopes observed in these studies are consistent with SFD slopes found for secondary craters across the solar system (Shoemaker 1965; Bierhaus et al. 2005; McEwen et al. 2005; Strom et al. 2011).

In as-yet unpublished work, Robbins has examined $\approx 6 \%$ of the lunar surface, $0-90^{\circ} \mathrm{W}$ by $0-33^{\circ} \mathrm{N}$, for secondary versus primary crater populations, similar to the Mercury study briefly described in the previous section. This region is mostly maria and includes the large and prominent Copernicus, Kepler, and Aristarchus craters. From a complete crater database of $D \geq 1 \mathrm{~km}$ craters $(\approx 30,500$ in this area) (Robbins 2016 ), there is an upturn, similar to that observed on Mercury, in the SFD for craters $D<\approx 4 \mathrm{~km}$. After removing morphologically obvious secondary craters from the population, the upturn in slope is at half that diameter, $D \approx 2 \mathrm{~km}$. Robbins and Hynek (2014), which is discussed in more detail in the next section, found similar results at Mars. Robbins and Hynek (2014) concluded that this upturn at $D \approx 2 \mathrm{~km}$ is likely an artifact of an incomplete separation of primary and secondaries with $D<\approx 2 \mathrm{~km}$, such that the steeper $D<\approx 2$ branch is due to a mixture of primary craters and "background" secondaries. The ongoing work of the Robbins lunar study will help determine whether a similar conclusion is appropriate for the Moon.

After several decades of research, we find it remarkable that the groundbreaking work of Shoemaker (1965) identified key results that remain important to the present day, and which provide the foundation for discussion and analysis in the remainder of this paper.

1. Fresh primary craters have a distinctively different morphology than fresh secondary craters that appear close to their parent primary.

2. Distant secondaries become more widely separated, more circular, and increasingly difficult to distinguish from primaries.
3. There are large numbers of shallow circular craters that cannot be assigned to either primary or secondary origin based on morphology.

4. Secondary craters have a very steep SFD, much steeper than known primary craters larger than $1 \mathrm{~km}$, so there should be a crossover diameter below which secondaries are more abundant than primaries.

One of the most striking recent results is the observation of 222 small primary impact craters (between a few meters and $40 \mathrm{~m}$ diameter), and $5 \times 10^{4}$ new dark and bright spots from before and after Lunar Reconnaissance Orbiter Camera images (Speyerer et al. [2016], which extends the work of Robinson et al. 2015). They estimate $\sim 10^{5}$ new spots form each year, largely from far-flung impact ejecta, although a large fraction of them may originate from meteoroid streams (McEwen et al. 2017b). In most cases, craters cannot be resolved in these spots, but the spatial patterns and association with new primary craters in some cases indicate they are from the impact of ejecta. Their observations, detecting of order $2 \times 10^{2}$ primaries and $1-5 \times 10^{4}$ secondary craters (or ejecta impact sites without craters) over the same time period, are the first direct estimate of the relative accumulation of primary and secondary craters on the Moon, albeit at small diameters. Also, this result validates the expectation that high-velocity impacts on medium-gravity planetary bodies should produce widespread secondaries (Shoemaker 1965; Bierhaus et al. 2012). Because small primaries generate fewer secondaries (both clustered and spatially random) than larger primaries (see the New Results section), it seems reasonable to expect the contrast between the relative number of primaries and secondaries will increase as larger craters form and broadcast their secondary population.

\section{Martian Data}

The impact crater records-both primary and secondary craters - of Mars have been interpreted through the lens of the Moon (Hartmann and Neukum 2001; Ivanov 20012001; Neukum et al. 2001). The presence of an atmosphere and active geology complicate small-crater analysis on Mars-small craters, including secondaries, are most affected by the ongoing erosional processes (see review by Williams et al. 2018). Small craters on the Moon and Mars also fade with time; however, their erosion (since volcanism ended on these planets) results from ongoing impact gardening from subsequent cratering events. Because of its atmosphere and active volatile transport each year, small crater erasure is more complicated on Mars, occurring at different rates depending on local wind, local volatile content, and underlying geologic materials. 
Many studies in the past decade use the standard production functions to assign ages to geologic regions or units on Mars (a small sampling includes Werner and Tanaka 2011; Goudge et al. 2012; De Pablo et al. 2013; Rodriguez et al. 2014; Baker and Head 2015; Landis et al. 2016), while a smaller set of papers address the effect that secondaries have on the crater populations and derived ages; we focus on that smaller set of papers here.

The McEwen et al. (2005) observations of the secondary crater population from the young, $\sim 10 \mathrm{~km}$ primary-crater Zunil reset the stage for contemporary discussion of Martian secondaries. Based on their observations of Zunil secondaries, they proposed that most Martian craters smaller than some crossover diameter are secondaries rather than primaries, and are not reliable for chronology. Tornabene et al. (2006) analyzed other rayed craters on Mars and found a spatial correlation between rays and clustered secondaries. The ray systems ranged from tens to nearly $10^{3} \mathrm{~km}$ in length, with a general trend to increasing ray length for increasing primary-crater size, though postformation processes can erase portions of the ray system. Their results show that, as on the Moon, the formation of a primary will distribute a population of secondaries far beyond the immediate neighborhood of the parent primary; this is true for both recognizable secondaries (those in clusters and rays) and those that are spatially random.

Hartmann (2007) compared the observed smallcrater $(<30 \mathrm{~m})$ production rate from Malin et al. (2006) with the HPF and NPF. The NPF is $6-11 \times$ higher than the Malin et al. (2006) observed rate, while the HPF is 3-6x higher than the Malin et al. (2006) rate. In his 2007 paper, Hartmann was careful to describe that his production function does not assume primary craters only, rather it is representative of primary-plusbackground secondary crater accumulation. More recent monitoring of the current Martian impact rate (Daubar et al. 2013) provides a longer baseline, with results that are generally consistent with those in Hartmann (2007). (Our simulations [Fig. 5 and corresponding discussion] for the Moon suggest the inclusion of background secondaries in the standard production functions is likely an important contributor to the overestimate of production functions relative to observations of the direct impact rate for small craters.)

Werner et al. (2009) used observations and theoretical arguments, drawn in part from Soderblom et al. (1974) and König (1977), to estimate the effect of secondaries on the overall observed crater SFD at Mars. The objective of the Werner et al. (2009) analysis was to determine at what sizes, and over what time scales, secondaries might degrade a surface age estimate derived from a crater-based chronological system. They concluded that while secondaries eventually are more numerous than primaries smaller than a certain diameter, that condition does not occur for wellresolved (by Mars Express) crater populations until a surface reaches ages well in excess of $3 \mathrm{Ga}$. They reiterated the finding of Neukum (1983) that the crossover diameter - the diameter at which secondaries become more numerous than primaries-should move to ever larger diameters as a surface ages and accumulates larger craters, which in turn generate larger secondaries. They argued that the consistency of the $\sim 1 \mathrm{~km}$ diameter transition from shallow to steep, regardless of surface age, is evidence that secondaries do not substantively impact the overall crater population. They also utilized the Soderblom et al. (1974) derivation that the slope of the secondary crater population from a single primary crater bounds the slope of the entire secondary crater population. In subsequent sections, we discuss new work that describes both the dependence of the secondary SFD on the primary SFD, and that the crossover diameter cannot migrate to arbitrarily large sizes. Our results suggest that the long-standing finding of Neukum (1983) (who suggested that the crossover diameter can migrate to large sizes) is not a strong constraint on the secondary population, and the secondary population from a single primary cannot be representative of the secondary population as a whole, contrary to the Soderblom et al. (1974) assumption.

Several recent studies examining Martian secondary impact craters were also done by Robbins and Hynek (2011a, 2011b, 2014). Lyot is $\approx 220 \mathrm{~km}$ in diameter and is the largest "young" impact crater on Mars (age estimates typically put it at early Amazonian to late Hesperian [Werner 2008; Robbins et al. 2013]). The researchers found that secondary craters in tight clusters from this impact could be plausibly traced as far as $5000 \mathrm{~km}$ across the planet. Examining the SFD of secondaries on the continuous ejecta, they found steep cumulative slopes of -5.6 , and within the distant clusters, the average cumulative slope was -3.9 (Robbins and Hynek 2011a).

A broader study by Robbins and Hynek (2011b) focused on examining the near-field morphologically identifiable secondary crater populations of 24 primary Martian craters with diameters $19.3-222 \mathrm{~km}$. From the study of nearly 13,000 secondary craters, they found that the SFD slopes around these primaries varied significantly from -3.3 to -8.0 , a wide range that suggests material differences - or other properties - can result in significantly steeper slopes than previously observed. In addition, they performed an area study and showed that, on average, the spatial density of secondary craters peaked approximately 2.4 primary 
crater radii from the rim of the primary, and that the radial distribution was approximately Gaussian. They also showed that this holds over $\approx 2$ orders of magnitude in primary crater size, even if there are nonobvious secondary craters: The spatial density of all craters around moderately well-preserved primary craters peaks at $\approx 2.4$ primary crater radii, indicating that even if they are not obvious morphologically, secondary craters can still be present and revealed through a spatial statistics argument. The authors extrapolated that if just 30 large, Holden-sized craters occurred uniformly across Mars' surface (Holden is a $\sim 150 \mathrm{~km}$ diameter crater in their study), $D<5 \mathrm{~km}$ craters would be mostly secondary craters across the entire Martian surface, and there are $\approx 95$ Holden-sized and larger craters on Mars. As demonstrated in Bierhaus et al. (2012), and which we explore further in the New Results section, the spatial relationship between a primary crater and its secondary population is a function of surface gravity and the minimum impact speed necessary to form a secondary, so the observed spatial relationships observed on Mars likely are not applicable to cratered surfaces in general.

Robbins and Hynek (2014) studied the global secondary crater population through the methods introduced in the previous sections on Mercury and the Moon, though in this published work, the effort was global and based on a $D \geq 1 \mathrm{~km}$ crater database (Robbins and Hynek 2012). They examined craters on the planet individually and determined the subjective likelihood that an impact was primary or secondary. Only craters that displayed classic morphologic criteria of secondary impact craters were marked as secondaries, which means that the results will be conservative: the results will, by design, miss "less obvious" secondary craters and treat them as primary craters by default. Even with this conservative approach, 72,208 craters with $D \geq 1 \mathrm{~km}$ were classified as secondary, while 308,998 with $D \geq 1 \mathrm{~km}$ were classified as primaries. They found, as one might expect, that the distribution of these "obvious" secondary craters was nonuniform, concentrated around younger, large primary craters (with the young, large Lomonosov and Lyot pair dominating). However, they found that if one lowers the threshold for at what point secondary craters are considered a "significant" contaminator of primary craters - e.g., when they are equal in number versus when secondaries comprise $25 \%$ of the total population-secondaries become an important component of crater populations over large areas of the planet for $\sim \mathrm{km}$-scale crater studies.

Most recently Quantin et al. (2016) examined the secondary-crater population from the primary crater Gratteri. They estimated that $\sim 1 / 2$ of Gratteri secondaries are concentrated in clusters resident in $\sim 2 \%$ of the surface around Gratteri (within $400 \mathrm{~km}$ ), with the remaining secondaries scattered in $98 \%$ of the surface. They present the view that secondaries cause uncertainty in age for surfaces $<10-20 \mathrm{Myr}$ old, but that older surfaces are less affected by secondaries: as a surface accumulates more spatially random secondaries, the existing spatial density is not as affected by another exposure to a shower of secondary craters. They conclude that surfaces between $10 \mathrm{Myr}$ (or $20 \mathrm{Myr}$ ) to $100 \mathrm{Myr}$ are amenable to dating by impact craters. Interestingly, they do not consider the effects of larger impacts as a surface remains exposed for longer durations, accumulating secondaries from larger, lessfrequent primaries. Over longer time scales, primary impacts larger than Zunil deposit a greater number of secondaries in one region than another. (As we will discuss in the New Results section, the ability to constrain an age, given a crater number density, can be no better than the spread of possible crater number densities for that age. As time passes, secondaries contribute to the spread in allowable number densities on a surface, thus affecting the uncertainty in an age estimate. Although we do not simulate Mars in our results reported here, nevertheless, all plots in Fig. 5 show consistency at least in that the variability in the total number density of craters is quickly dominated by secondaries.) Finally, Hartmann and Daubar (2017) examined young terrains on Mars, in conjunction with direct observations of newly formed craters on Mars, and found that the HPF overpredicts the production rate of small craters by a factor of $4-8$.

\section{Current/Past Impact Rates on Terrestrial Planets}

Before we move to observations of the outer solar system, we first address a topic relevant for the inner solar system, namely direct observation of the current impact rate. One reason the relative abundance of primaries versus secondaries has been in debate is because we did not know the formation rate of small primary craters. Observations of Mars spanning more than a decade (Malin et al. 2006; Daubar et al. 2013, 2015) and several years at the Moon (Speyerer et al. 2016) are now providing useful estimates of the current impact rate at which very small primary craters $(<50 \mathrm{~m})$ form.

The observed new crater SFD on both Mars (for $D>4 \mathrm{~m}$, Daubar et al. 2013) and the Moon (for $D>7 \mathrm{~m}$, Speyerer et al. 2016), especially their Extended Data fig. 5), are shallower than the NPF and HPF, which leads to a discrepancy between the model crater production functions and the SFD of recently formed craters. Speyerer et al. (2016) fitted a subset of their lunar data above the completeness limit, from 10 to 
$20 \mathrm{~m}$, and from that subset derived a steeper SFD than the NPF, and estimated an impact rate for these diameters that is $33 \%$ higher than the NPF. We note that a fit to the entire data set above the Speyerer et al. (2016) completeness limit (i.e., a fit to all craters $>7 \mathrm{~m}$ diameter, rather than only those between 10 and $20 \mathrm{~m}$ ) would result in a slope more shallow than the NPF. Interestingly, observations of Earth bolides (Brown et al. 2013) are also shallower than the crater-scaled sizes from lunar production functions. This suggests that the NPF and HPF include a contribution of background secondaries in their derivation of the smallcrater formation rate-inclusion of these background secondaries steepens their production rates relative to that observed. However, the completeness limits on new crater discoveries both at Mars and the Moon may comprise fuzzy diameter boundaries, flattening the slopes even at diameters for which craters are well resolved. Another possibility is that the SFDs for both primaries and secondaries are similar in this size range. Secondaries at these diameters are made by ejecta from a range of primary-crater diameters, and the majority of the secondaries could be from larger (and thus lessfrequent) primary craters. We know that at larger diameters, primaries have varying SFDs. Because of the relationship between primary and secondary SFDs (see later discussion), secondaries in the $<20 \mathrm{~m}$ size range are a mixture of SFDs as well, and on average will be less steep than the secondaries made by an exclusively steep primary SFD.

The observations of the actual crater production rate sidestep many of the problems that plague crater analyses (distinguishing primaries from secondaries, inferring an impactor diameter, attaching a formation rate to observed craters to invert a crater density into an age estimate), but there are two caveats to keep in mind. First, what is not known is whether the impact rate in the most recent decades is typical of geologic time. Many workers have assumed a constant impact rate over the past $\sim 3 \mathrm{Ga}$ (e.g., Neukum et al. 2001). Some workers have suggested that impact rates in recent geologic time have increased (McEwen et al. 1997; Culler et al. 2000; Mazrouei et al. 2015), have declined (Hartmann et al. 2007; Quantin et al. 2007; Robbins 2014), or have fluctuated (e.g., Bottke et al. 2007; Kirchoff et al. 2013). However, the current impact rate has varied over time; secondary crater production must follow the same pattern. The second caveat is that the diameter range for the observed, newly formed impact craters is only from a few meters to a few tens of meters (for diameters thought to be complete). We say "only" here because the diameter range of relevance for secondaries is roughly three orders of magnitude (from a few meters to a few $\mathrm{km}$ ). We hope for the continued long life of existing orbiters, and the introduction of new orbiters that will permit us to extend the baseline observations of the actual impact rate to longer time scales, and thus presumably to include larger impactors.

\section{Icy Satellites}

Though Cassini has been the only source of new high-resolution image data of icy satellites in the past decade, ongoing analysis of Galileo data also has provided new results for icy surfaces. In addition to the widespread clustered craters on Europa interpreted as secondaries (Bierhaus et al. 2005), secondary craters from a few specific primaries on Europa are traceable to their parent primary. The large impact structure, Tyre $(D \sim 38 \mathrm{~km})$, has the best radial image coverage at resolutions acceptable for mapping secondary craters, as well as a higher resolution inset (Bierhaus and Schenk 2010; Singer et al. 2013). Secondaries from the brightrayed crater Pwyll $(D \sim 27 \mathrm{~km})$ can be mapped up to $\sim 1000 \mathrm{~km}$ away in the few small high-resolution images taken over a prominent ray (Alpert and Melosh 1999; Bierhaus et al. 2001). On Ganymede, prominent secondary fields are observed around the large impact basin Gilgamesh $(D \sim 585 \mathrm{~km})$ and the central peak crater Achelous $(D \sim 35 \mathrm{~km})$, the latter of which formed on younger, resurfaced grooved terrain. Singer et al. (2013) used all of these secondary fields to derive icyejecta size-velocity distributions and SFD slopes, finding generally steep SFDs, and a relatively rapid decrease in maximum secondary diameter as a function of range from the parent primary. In addition, the image coverage on Ganymede allows identification of secondaries originating from other primary craters, although only for limited areas around the primary (Hoogenboom et al. 2015). Furthermore, even larger craters on Ganymede's bright terrain $(D>1 \mathrm{~km})$ may be dominated by secondaries as indicated by their significant spatial clustering (Kirchoff, 2018).

An interesting contrast emerged from comparing the Galilean satellites with the Saturnian satellites. While adjacent secondary craters and secondary clusters are prominent on the Galilean satellites, they are largely absent on the Saturnian satellites. Rhea is the only Saturnian satellite to show an "obvious" set of secondary impact craters, characterized as a tight clustering of many small craters within, on the rim, and outside the east half of Inktomi crater $\left(66.4^{\circ} \mathrm{E}\right.$, $-11.9^{\circ} \mathrm{N}, \quad D \approx 49 \mathrm{~km}$ ) (Kirchoff and Schenk 2010; Hoogenboom et al. 2015; Robbins et al. 2015). The clustering and relatively steep SFD cumulative slope of $-3.9 \pm 0.2$ (which is near the average slope common to secondary craters in the inner solar system) are hallmarks of a secondary population. Bierhaus et al. 
(2012) developed a hypothesis to explain the contrast between the Galilean and Saturnian satellites, which we subsequently review in more detail. In brief, simple differences between the typical primary impact speed, and the surface gravity-which, when combined with $v_{\min }$ (the minimum impact speed necessary to form a secondary crater), defines the spatial relationship of secondaries to their parent primary-can have significantly different outcomes. The higher surface gravities of the Galilean satellites result in a spatial distribution of secondaries that is familiar relative to the observations from the terrestrial planets, whereas the lower surface gravity of the mid-sized Saturnian satellites causes a different geometry between a primary and its secondary population.

\section{Pluto and Charon}

New Horizons data returned from the Pluto system provided (1) new information on outer solar system primary impactor populations, and (2) created a new mystery, namely the lack of secondary craters on both objects. Smaller $(<\sim 10 \mathrm{~km})$ craters on Pluto and Charon show a shallow ( -2 differential or shallower) SFD for primary craters (Moore et al. 2016; Singer et al. 2016; Robbins et al. 2017), confirming that small Kuiper Belt Objects (KBOs) hitting Pluto and Charon-and thus members of the same transneptunian populations hitting the Saturnian and Jovian satellites-should have a shallow slope at this diameter range. Though low densities of secondaries on these bodies are predicted (Bierhaus and Dones 2015), there are no obvious secondary craters apparent on either Pluto or Charon. Image scales should be adequate to observe secondary craters (as good as $80 \mathrm{~m} / \mathrm{px}$ on Pluto and $160 \mathrm{~m} / \mathrm{px}$ on Charon), although higher resolution coverage is limited in areal extent. Degradation of surface features occurs relatively quickly on Pluto due to its seasonal sublimation and redeposition of frosts, and also due to active endogenic geologic processes. On Charon, however, there are broad surfaces with no obvious degradation mechanisms other than micrometeorite bombardment. Further research is needed to deconvolve the various circumstances (e.g., low primary impact velocities, surface materials, dynamical environments) that lead to the apparent dearth of secondary craters on Pluto and Charon. The apparent total absence of secondaries on these bodies, in contrast with the unique abundance on Mercury, suggests there is a relationship between impact velocity and the nature of material ejected during the formation of a primary impact crater.

\section{Self-Secondary Cratering}

The idea of self- or auto-secondary cratering, where fragments ejected from a primary impact may form secondary craters on the ejecta blanket of the parent crater, was introduced by Shoemaker et al. (1968) and has recently received more attention. We do not discuss the effect of auto-secondaries on a crater-based chronology; however, we briefly describe them in this paper for completeness. Further work in the field may raise important considerations for chronology-e.g., using superimposed small crater populations on the ejecta blankets of large craters to derive an age for the large primary impact. On several lunar primary craters, researchers found different crater densities on individual melt ponds both within and outside of the crater, and also across the ejecta blanket (Plescia et al. 2010; Plescia 2012; Zanetti et al. 2014, 2016; Van der Bogert et al. 2017); debate is ongoing about the importance of variations in target properties versus self-secondary cratering. An additional study concluded selfsecondaries are present around Hokusai crater on Mercury (Xiao et al. 2016). Furthermore, some misshapen, small $(<100 \mathrm{~m}$ diameter) craters may even have been formed on partially molten melt ponds or modified/partially filled by melt (Plescia and Cintala 2012; Williams et al. 2014a, 2014b; Zanetti et al. 2015). These observations imply some small craters formed after the ejecta blanket but before, or shortly after, the impact melt was emplaced.

However, the mechanism for this process is not obvious, in part because if they form in the same fashion as traditional secondaries, they require a unique and potentially rare combination of ejection angle and speed. It could be that self-secondaries result from highangle ejecta that are launched soon after the impact, at speeds just below escape velocity, and the ejecta take some time to reimpact the surface. For example, an ejecta fragment launched from near the center of the crater at a nearly vertical angle $\left(89.8^{\circ}\right)$ and just below the lunar Hill sphere escape velocity $\left(2.34 \mathrm{~km} \mathrm{~s}^{-1}\right)$ could reimpact Aristarchus' ejecta blanket (for this example we used $\sim 1.25$ times the crater radius) after a $40 \mathrm{~min}$ ballistic flight time (substantially longer than it takes the ejecta blanket to form). However, laboratory experiments show that most material is ejected at angles of $45^{\circ} \pm 15^{\circ}$ (Cintala et al. 1999; Anderson et al. 2003; Durda et al. 2012); indeed these experiments into granular media do not see ejection angles above $60^{\circ}$. Much, if not all, of the material near the impact point is vaporized or at least melted, rather than ejected as solid fragments (e.g., Melosh 1989). At lower ejection angles, the velocity must also be lower in order to still impact the ejecta blanket, and thus the flight times are shorter. A fragment ejected at $80^{\circ}$ would impact the same point in the ejecta blanket as the above example if launched at a velocity of $345 \mathrm{~m} \mathrm{~s}^{-1}$, which would yield a 6.4 minute time of flight. Material spalled off the 
surface (Melosh 1984) could be ejected as a solid fragment at high velocity, and may have a different trajectory than the main excavation flow. Numerical experiments have found that more oblique $\left(>30^{\circ}\right.$ from normal) primary impacts may produce such high-angle spall fragments (Artemieva and Zanetti 2016).

The Sedan nuclear explosion is often invoked to argue that significant self-secondary cratering is possible. However, the explosives were buried $193.5 \mathrm{~m}$ deep and video of the event shows that the explosion domed up the surface by $\sim 90 \mathrm{~m}$. Extensional stresses must have created vertical fractures in this dome, and the video shows vertical jets of debris erupting from several locations. This event does not resemble an impact event for early-stage ejecta.

A second possibility-explored in an attempt to reconcile the differing crater densities on dense versus rubbly lava flows and ejecta versus melt ponds-is the role of different target materials which can form different crater sizes from the same impactor size (Dundas et al. 2010; Artemieva and Zanetti 2016; Van der Bogert et al. 2017). At Aristarchus on the Moon, where differing crater densities were observed across the ejecta blanket, there was an association of lower crater density with areas covered by more melt ponds (Zanetti et al. 2014). The size difference of $\sim 20 \%$ expected between craters formed on frozen melt ponds and a more porous ejecta unit from the same size impactor may not seem like it could account for the total magnitude difference in crater densities observed, but the steep SFD makes this effect significant to model terrain ages.

Although the details of auto-secondary formation are under development and are even less understood than nearby or distant secondaries, the differing crater densities are an intriguing observation. Because ejecta blankets of large impact craters are often treated as younger, fresh surfaces with a chronologically valuable superimposed small-crater population, it is important to understand the existence and magnitude of selfsecondaries (Plescia and Robinson 2015; Xiao et al. 2016).

\section{Effect on Crater SFDs and Impact-Crater Based Chronologies}

Dating of surfaces from impact-crater statistics relies on the basic assumption that there is a deterministic relationship between a surface age and a crater spatial density - a given crater density corresponds to a given age. The key question for the effect of secondaries on impact-crater chronologies is whether or not the accumulation of secondaries, on average, preserves the deterministic relationship between a certain number and sizes of craters within a surface and the age of that surface. On one hand, when millions of craters form essentially simultaneously as secondaries, this is an extreme violation of the requirement for statistical age determination. Fresh secondaries that are close to their source primary often are distinctive in morphology and in part can be excluded from crater counts, but distant secondary craters (sometimes called "(far-) field secondaries," or "background secondaries") can be difficult to distinguish from primaries because they are more circular and may be less obviously clustered. On the other hand, Quantin et al. (2016) and Hartmann (2007) argued that field secondaries can dominate the cratering statistics yet give useful chronology data, but the resulting error bars must be much larger than for a population of only primaries, and quantitative error bars have not been derived or published. Most workers agree that secondaries should be avoided, but debates persist about the reliability of crater-derived chronologies when secondary craters might be present.

Another important observation that we note is the evolution of the crossover diameter across the solar system. On Mercury, a steeper crater SFD occurs for crater diameters $\leq 10 \mathrm{~km}$; on the Moon, Mars, and the Galilean satellites, the steepening occurs for diameters $\sim<1 \mathrm{~km}$; on the Saturnian satellites (where steepening exists), it occurs for diameters $<1 \mathrm{~km}$; and on Pluto and Charon, secondaries do not seem to exist at all. There are obvious differences between these objects, including surface properties and impact speeds. The very high impact speeds on Mercury, along with the largediameter secondary population, is a compelling contrast with the absence of secondaries on Pluto and Charon, and the low mean impact speeds on those KBOs. In addition to the factors discussed later, the mean impact speed-and perhaps target properties-may have consequences for crater ejecta, and thus the resulting secondary-crater population (or its absence).

Even though secondaries can be as large as $\sim 5 \%$ of their parent primary, there are only a few of those biggest secondaries, and the vast majority of secondaries are typically smaller than the primary by $\sim 10^{2}$ to $10^{3}$. Thus, the largest craters on a world are safely assumed to be primaries. This basic fact sometimes gets lost in the discussion of crater-based chronologies-we fundamentally subscribe to the power and validity of crater-based chronologies when primary craters are the dominant population. However, small areas and young surfaces lack significant numbers of large craters so smaller craters must be considered, and small-crater populations are more likely to contain significant numbers of secondaries. In these cases we must understand the secondary effect. What seems the least controversial within the community is the generally 
"steep" SFD of secondaries (where "steep" is for $\alpha>4$, or equivalently, $b>3$ ), though as mentioned in previous sections, studies of individual secondary populations show a range of SFDs.

As described earlier, the "reference standard" crater population derives from decades of analysis of the lunar surface. Cumulative numbers of maria craters from $\sim 1-$ $100 \mathrm{~km}$ have exponent $\alpha \sim 2$, whereas those from $\sim 100$ $1000 \mathrm{~m}$ have $\alpha \sim 3.8$ (Hartmann et al., 1984). The idea that this change in slope at $1 \mathrm{~km}$ represented the crossover diameter between primaries and secondaries, citing Shoemaker (1965), has been often repeated in the cratering literature (e.g., Soderblom et al. 1974; Wilhelms et al. 1978; Carr 2006). However, the actual analysis of Shoemaker (1965) does not exactly match that simple summary.

From direct interpretation of the Ranger VII photographs, Shoemaker (1965) concluded "In the diameter range from $300 \mathrm{~m}$ to $1 \mathrm{~km}$... craters of this size are predominantly secondary." However, since secondaries are concentrated in identifiable rays (prominent in the Mare Cognitum regions imaged by Ranger VII), as discussed at length in Shoemaker (1965), the crossover diameter for areas between rays must be at a smaller diameter. Based on extrapolation of the telescopically observed primary craters $>1 \mathrm{~km}$ and a model for secondary crater generation from this population of primaries, he predicted that the crossover diameter would be "a little above $200 \mathrm{~m}$ " rather than $300 \mathrm{~m}$ to $1 \mathrm{~km}$. Furthermore, based on the terrestrial meteorite infall rate, Shoemaker concluded that the primary SFD had to become steeper than the extrapolation for craters $<1 \mathrm{~km}$ diameter, so the crossover diameter at which secondaries exceeded primaries must be at an even smaller diameter.

A major advance in understanding the steepness of the production function for small primary craters on the Moon and Mars has come from direct observation of new impacts (Malin et al. 2006; Daubar et al. 2013; Speyerer et al. 2016). If the impactor SFD that makes small craters (roughly $<1 \mathrm{~km}$ ) in fact maintained the same slope (cumulative exponent $\alpha \sim 2$ ) as $1-100 \mathrm{~km}$ craters (as suggested most recently by Xiao and Strom 2012), then the cumulative number of new craters $\geq 10 \mathrm{~m}$ diameter should be $10^{4}$ times less than predicted by the Hartmann and Neukum functions. Instead, the numbers of new craters at $\sim 10-20 \mathrm{~m}$ express an SFD that is similar to the production functions at that size range. From these data on hundreds of new impacts, it is now clear that the lunar and Martian primary production functions for craters in the diameter range observed ( $\sim 5-50 \mathrm{~m}$ diameter) must be steeper than for larger craters, unless today's impact rate is not typical of geologic time. If the production function has $\alpha \sim 2$ to small size craters, then the new crater data would require that the current cratering rate is $\sim 10^{4}$ times greater than the long-term average. In the absence of any other evidence for such a dramatic recent change, such as a new asteroid family, we conclude that this is highly unlikely.

In contrast, the production function may be much shallower in the outer solar system where the impactor population is dominated by comets rather than asteroids (Zahnle et al. 2003). Bierhaus et al. (2012) suggested that the primary production function has $\alpha \sim 1$ on the icy Galilean and Saturnian satellites for crater diameters smaller than a few tens of $\mathrm{km}$, an observation that received strong support from the New Horizons flyby of Pluto and Charon, whose surfaces also show a shallow crater population with $\alpha \sim 1$ for diameters $<10 \mathrm{~km}$ (Singer et al. 2016; Robbins et al. 2017). Even if the impactors striking Pluto and Charon are not the same mix of small-body reservoirs as those hitting the satellites of the giant planets, there is overlap between the populations (Bierhaus and Dones 2015; Greenstreet et al. 2015).

Why do secondaries and small primaries produce similarly steep production functions in the inner solar system? Hartmann (1995) suggested this could be because small asteroids impacting the planets are in fact secondary fragments from impacts on asteroids, so the fragments have the same SFD as high-velocity ejecta from impacts on planets. However, ejecta has a strong size-velocity anticorrelation (Vickery 1987; Singer et al. 2013, 2015), so small fragments reimpact at higher velocities than larger fragments. This has the effect of greatly steepening the SFD of secondary craters (McEwen and Bierhaus 2006; see their fig. 7). Ejecta from asteroids impact planets with velocities that have no relation to fragment size, so the SFD of small primary craters would not be expected to be as steep as that of secondaries, unless the SFD of small asteroids is very steep for some other reason. Some explanation other than that of Hartmann (1995) is needed. Possibly smaller rocks ejected from asteroids are more likely to reach resonances sending them to Earth, either due to higher ejection velocity (e.g., Cellino et al. 1999) or the Yarkovsky effect (Farinella et al. 1998; Bottke et al. 2006).

Where does all of this leave us for chronology using small craters? The answer is not straightforward because the magnitude of secondaries depends on the age of the surface, the impacting population, how many secondaries are made for a given impact, and how they are distributed over the surface. We discuss this in more detail in the Consequences for Crater SFDs and Age Determination section. 


\section{Scaling Laws and Ejecta Mechanics}

The consequence of secondaries to the local, regional, and global crater populations depends on their production and distribution. Crater scaling laws, derived from impact experiments, observations of craters, and modeling all describe the physical relationships between impactor, target, and resulting crater. Researchers often use the scaling laws to connect impactor diameter to crater diameter, assess target strength, or examine the evolution of ejecta during the excavation process.

\section{Mechanics of Excavation and Target Properties}

As the impact shock and rarefaction waves travel through a planetary surface, material is ejected both through a bulk excavation flow, which creates approximately the upper half of the transient crater cavity, and through spallation of near-surface material from stress-wave interference (e.g., Melosh 1989). Spalls are unique ejecta fragments in that they are launched at high velocity but low shock pressures (Melosh 1984, 1989). Spalls may form distal secondary craters or even be ejected off of the parent planetary body. However, spall fragments should make up only a small part of the overall mass of ejecta. In the main excavation flow, intact fragments launched from closer to the impact point are subjected to higher shock pressures, and as a result are smaller fragments with higher velocities compared with those launched farther from the impact point. This physical process results in larger secondary craters near the primary, and smaller or comparably sized secondary craters farther from the primary. Secondary craters do not get larger with range as one might expect given the higher impact velocity, because the high-velocity fragments are smaller (Vickery 1986).

Dynamic fragmentation of material, such as that occurring during an impact, has been studied through laboratory experiments, field experiments such as explosion testing, analytical and numerical modeling, and empirical studies. In impact science, Grady-Kipp fragmentation has been used to predict the "characteristic" or most common size of fragments from the dynamic breakup of coherent rock or ice (Grady and Kipp 1985; 1987; Melosh 1989, chapter 6; Grady 2008). Ramesh et al. (2015) presented a summary of impact-relevant dynamic fragmentation research to date and highlighted where new studies would advance this field. The question of how these mechanisms can be scaled from laboratory settings to planet-wide processes is still a topic needing active research. Empirical studies can complement other lines of inquiry on dynamic fragmentation.

Bart and Melosh (2010a, 2010b) measured boulder diameters and distances from their source craters for several lunar primary craters. Eight of the 10 craters they examined have diameters $<1 \mathrm{~km}$, one crater is $\sim 4 \mathrm{~km}$ diameter, and one crater is $\sim 27 \mathrm{~km}$ diameter. For the $<1 \mathrm{~km}$ craters, they found that the presence of the fine-grained lunar regolith decreases the volume of ejecta blocks, as the excavation flow first must eject the overlying regolith before reaching the more competent rock below. They fit their data to the relationship $\left(d_{\max }=A v_{\mathrm{ej}}^{-\beta}\right)$, where $d_{\max }$ is the largest boulder diameter ejected at velocity $v_{\mathrm{ej}}$, and $A$ and $\beta$ are fit to the data. They found $\beta$ ranging from 0.5 for the smallest craters to almost 4 for the largest crater. When they include the data from Vickery (1987), there is a trend of increasing $\beta$ with increasing crater size, suggesting a scale-dependent effect for the excavation flow, or at least the late-stage excavation flow.

Similar studies used secondary craters to estimate ejecta fragment sizes and velocities (e.g., Vickery 1986, 1987; Singer et al. 2013, 2014), which in turn constrain ejecta fragment size-velocity distributions. In these papers, the authors measured the diameter of secondary craters around their parent primary and used scaling laws to estimate the fragment diameter that made the secondary. The Singer et al. results expand the Vickery results, and provide data for icy surfaces, to define the $\left(d_{\max }=A v_{\mathrm{ej}}^{-\beta}\right)$ relationship of crater ejecta. The Singer et al. data also suggest that $\beta$ increases (and thus $-\beta$ decreases) for larger diameter primary craters.

Although there is scatter in the relationship, the consistent trend seen in the Vickery data, Bart and Melosh data, and the Singer et al. data demonstrate the inverse velocity relationship for crater ejecta, as well as scale-dependent behavior of $\beta$. The increase of $\beta$ as primary-crater diameter increases suggests that the largest ejecta blocks from the largest craters are hard to make, or preserve in flight, or both. This may have to do with pre-existing fractures or the absence of large, competent material in the surface and near-surface of regolith and mega-regolith. This observation may help explain why the crossover diameter does not always migrate to progressively larger sizes as a surface ages. Although larger primary craters generate larger secondaries, there are only a handful of those maximum-sized secondaries, not enough to impact the overall SFD at the size. (Plus, many researchers avoid "obvious secondaries" in their crater measurements, and at least on the terrestrial planets, the very largest secondaries are typically part of the obvious population.) Because of the primary-crater diameter dependence of $\beta$, the fragment diameters also decrease more quickly for the larger primaries. If generally true, then large primary craters may not generate enough secondaries to impact the overall crater SFD at diameters near, but still smaller than, the largest 
secondary. This behavior would limit the migration of the crossover diameter to progressively larger sizes.

\section{Scaling Laws and Mass to Make Secondaries}

Impact crater scaling laws use impactor properties (diameter, density, mass, impact velocity) and target properties (strength, density, surface gravity) to estimate a number of characteristics of the resulting crater. A common parameter of interest is the crater diameter; however, scaling laws also provide quantitative relationships between ejecta mass and ejecta speeds. Housen and Holsapple (2011) provided a comprehensive description of impact crater experiments, and the resulting scaling fits, with special attention to crater ejecta. Because secondary craters are the result of crater ejecta, the Housen and Holsapple (2011) work provides a mechanism to explore the formation of secondary craters.

In particular, Bierhaus et al. (2012) used scaling laws outlined in Housen and Holsapple (2011) to explore the variation of secondary-crater production as the conditions of an impact change, using the Saturnian and Galilean satellites as a test case to evaluate the theory. Bierhaus and Dones (2015) expanded the analysis to include Pluto and Charon. In brief, the mass available to make secondary craters depends on the primary crater population, and the inherent variations of just a few parameters (surface gravity $g$, impact speed $U$, and escape velocity $v_{\text {esc }}$, they assumed a constant $45^{\circ}$ impact angle). Because these parameters are different between worlds, the resulting primary and secondary crater populations can be different even if the impacting population is the same. The importance of primary impact velocity for the resulting crater ejecta velocity distribution is consistent with results in fig. 5 of Artemieva and Ivanov (2004), which shows an expanded volume of higher velocity ejecta for a $35 \mathrm{~km} \mathrm{~s}^{-1}$ impact relative to a $10 \mathrm{~km} \mathrm{~s}^{-1}$ impact.

Another important parameter introduced in Bierhaus et al. (2012) is $v_{\min }$, the minimum speed necessary to form a secondary crater; this parameter is not part of scaling laws but fundamental to whether and how many secondary craters form on a surface. Above some speed, ejecta blocks form secondary craters rather than landing intact or breaking up and making a rough surface with no obvious crater. The velocity range between $v_{\min }$ and $v_{\text {esc }}$ defines the range of ejection velocities that form secondary craters (see Fig. 2). Note that there can be cases for which $v_{\mathrm{esc}}<v_{\min }$, in which case no secondary craters form on that object, in theory.

Using the relationships defined by scaling laws, in conjunction with $v_{\min }$, it is possible to estimate the amount of mass available to make secondaries for a

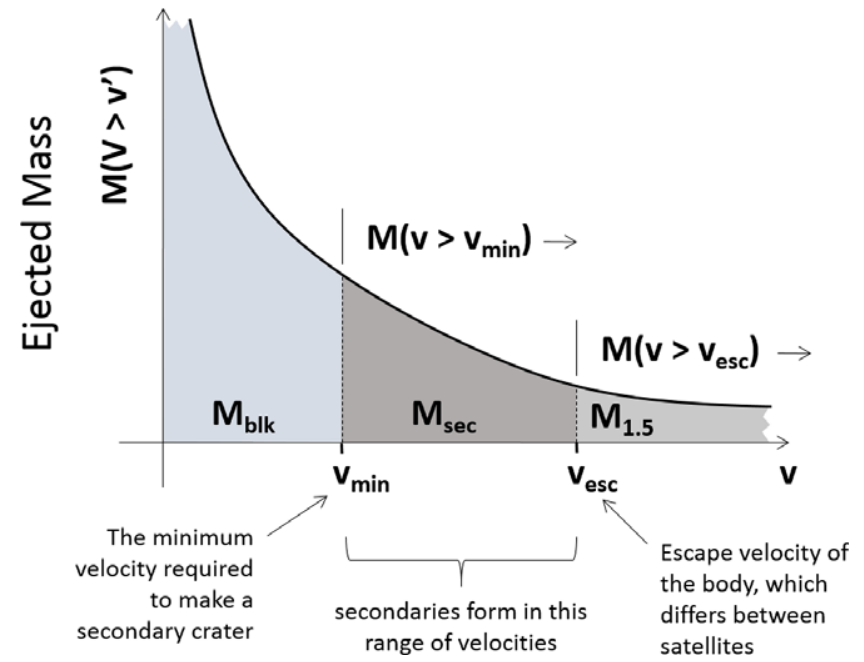

Fig. 2. A schematic illustrating ejected mass as a function of ejection velocity. Less mass is ejected at higher velocities. Ejected mass can be grouped into three basic categories: (1) mass moving less than $v_{\min }$ (the minimum speed necessary to form a secondary crater), most of this mass goes into the ejecta blanket; (2) mass moving faster than $v_{\text {min }}$ but slower than $v_{\text {esc }}$, this is the mass available to make secondary craters; and (3) mass moving faster than $v_{\text {esc }}$, which is mass available to make sesquinary craters. Figure after Bierhaus et al. (2012). The value of $v_{\min }$ will vary between target types, and $v_{\text {esc }}$ between different objects, and thus the mass available to make secondary craters for a given impact will be different between target objects. (Color figure can be viewed at wileyonlinelibrary.com.)

given, single-sized impactor. Bierhaus et al. (2012) provided the analytical steps, and applied them to the Saturnian satellites using a $1 \mathrm{~km}$ diameter comet; Bierhaus and Dones (2012) applied the analysis, using a $1 \mathrm{~km}$ asteroid, to the inner solar system: Mercury, the Moon, Mars, and Vesta. In brief, the methodology estimates the mass ejected between the two limiting velocities $\left(v_{\min }\right.$ at the lower limit and $v_{\text {esc }}$ at the higher limit) via the equation

$$
\frac{M(v>\hat{v})}{m_{\mathrm{i}}}=C_{4}\left(\frac{\hat{v}}{v_{\mathrm{i}}}\right)^{-3 \mu}\left(\frac{\rho_{\mathrm{t}}}{\rho_{\mathrm{i}}}\right)^{1-3 v}
$$

where $C_{4}, \mu$, and $v$ are constants; $M(v>\hat{v})$ is the mass ejected faster than velocity $\hat{v} ; v_{i}$ is the impactor velocity (scaled by $\cos \left(45^{\circ}\right)=0.71$ to account for an average impact angle); $\rho_{\mathrm{t}}$ is the density of the target; and $\rho_{\mathrm{i}}$ is the impactor density. The mass available to make secondaries is $m_{\mathrm{sec}}=M\left(v>\hat{v}_{\min }\right)-M\left(v>\hat{v}_{\mathrm{esc}}\right)$; see graphic in Fig. 2. Table 2 summarizes the results of both studies, normalizing the mass available to make secondaries $\left(m_{\mathrm{sec}}\right)$ by the impactor mass $\left(m_{\mathrm{i}}\right)$. There are striking trends from this analysis

1. Mercury should express the most significant secondary population of the inner solar system, and 
Table 2. A list of solar system objects with zero or low atmospheric densities, and the ratio of $m_{\mathrm{sec}}$, the mass available to make secondaries, to $m_{\mathrm{i}}$, the mass of a single impactor. The impactor is a $1 \mathrm{~km}$ diameter asteroid for the inner solar system objects, and a $1 \mathrm{~km}$ diameter comet for the outer solar system objects. The values for rocky surfaces (Io, and inner-solar-system objects) assume $v_{\min }=250 \mathrm{~m} \mathrm{~s}^{-1}$, and the values for icy outer-planet satellites assume $v_{\min }=150 \mathrm{~m} \mathrm{~s}^{-1}$.

\begin{tabular}{lr}
\hline Object & $m_{\mathrm{sec}} / m_{\mathrm{i}}$ \\
\hline Inner solar system & \\
Mercury & 10.0 \\
Moon & 4.1 \\
Mars & 1.2 \\
Vesta & 0.3 \\
Jovian satellites & \\
Io & 167.3 \\
Europa & 4.6 \\
Ganymede & 3.2 \\
Callisto & 2.2 \\
Saturnian satellites & \\
Mimas & 0.0 \\
Enceladus & 1.5 \\
Tethys & 2.4 \\
Dione & 2.4 \\
Rhea & 2.0 \\
Iapetus & 0.5 \\
\hline
\end{tabular}

maybe the most significant secondary population in the solar system. This is due to the very high primary impact speed(s), which generate significant amounts of fast-moving ejecta, and the high escape velocity, meaning much of the ejecta are retained to make secondary craters. This prediction is borne out by observations of crater populations seen in MESSENGER image data (Strom et al. 2008, 2011)

2. Io, the Jovian satellite with frenetic volcanic activity, has the highest value of $m_{\mathrm{sec}} / m_{\mathrm{i}}$ for any object in the solar system, over an order of magnitude larger than Mercury. Io's value results from the same combination of parameters that lead to the relatively large result for Mercury: high impact speeds, and an escape velocity high enough to retain much of that fast-moving ejecta. Other factors contribute to Io's increase over Mercury: Io's lower surface gravity leads to larger craters, and the bulk density difference between a typical comet and asteroid means the normalization factor $\left(m_{\mathrm{i}}\right)$ is smaller for Io. An amazing feature of Io's surface, as seen from all missions with resolved images of Io to date, is that the surface is crater free (at resolutions of available data), so we are unable to compare this estimate against observations. (A related analysis by Zahnle et al. [2008] predicts that Ionian primary impacts should produce sesquinary craters on other Jovian moons, especially Europa.)

3. An impactor of a given size will make more secondaries on the Moon than on Mars because the faster mean primary impact speed on the Moon, and lower surface gravity, results in a larger transient primary crater on the Moon-and thus more mass is available to make secondaries.

4. Although Vesta should have fewer secondaries per impact, by a factor of several, than Mars, and $\sim 10 \times$ less than the Moon, the value is nonzero. Given the relatively low surface gravity of Vesta (compared with the Moon and Mars), more of the secondary population from each primary impact is globally distributed (not unlike our results for Enceladus, see the New Analysis section). The expected presence of secondaries on Vesta is confirmed by Dawn observations (Neesemann et al. 2014).

5. For the Galilean and Saturnian icy satellites, Europa should have the most secondaries per impact. This is because a high mean impact speed (and thus large primary impact crater), and relatively large escape velocity (compared with the Saturnian satellites), result in the most mass available to make secondaries for these bodies.

6. If $v_{\min }$ is of order $150 \mathrm{~m} \mathrm{~s}^{-1}$ for icy surfaces (the value used in the analysis), then Mimas does not have secondary craters, because $v_{\min }>v_{\text {esc }}$. (The velocity to reach the Hill Sphere of Mimas is $\sim 130 \mathrm{~m} \mathrm{~s}^{-1}$, which effectively is the escape velocity, see Bierhaus et al. [2012] and Alvarellos et al. [2005].)

7. Tethys, Dione, Rhea, and Callisto should have comparable secondary populations (by mass), though their spatial distributions will be different.

A caveat to this analysis relates to the role of impact melt: the calculations summarized above do not account for how much of the ejected mass is melted material. Melosh (1989) described an impact melt model that depends on gravity and impact velocity, thus the same-sized primary crater will generate different amounts of melt on different target bodies. While ultimately impact melt plays a role in the fraction of mass available to make secondaries, there are clear dependencies between impact velocity, surface gravity, ejection-velocity distributions, and escape speed that we have considered.

Ultimately, impact melt caveat included, this analysis demonstrates that one must consider the specifics of each object in regard to the magnitude of the secondary population and the consequences to crater SFDs and associated analyses (e.g., deriving 
surface ages). The New Analysis section matures these earlier studies to resolve the bulk mass differences into actual secondary crater populations.

\section{0}

We briefly address Io because of its prominence in Table 2: Io theoretically has considerably more mass than any other body to make secondary craters for a given impact. (This assumes that the impact speed distributions are approximately correct, and that the scaling law estimate for $M_{\mathrm{sec}}$ at least preserves the relative magnitude between objects, even if the absolute value is incorrect.) Zahnle et al. (2008) and Alvarellos et al. (2008) considered a related calculation, specifically that fast-moving impactors striking Io should generate large quantities of ejecta that escape Io to hit Europa (and other moons). (However, their prediction for the number of Ionian sesquinaries on Europa does not match observations - they predict that Ionian sesquinaries on Europa are nearly as abundant as native Europan secondaries, but observations show clustered secondaries dominate Europa's crater population [Bierhaus et al. 2005]). Nevertheless, Io ejecta should reach Europa.

To date it has been impossible to test this hypothesis because no impact crater is visible in any images of Io. Of course, Io lacks significant moderateor high-resolution image coverage, and so the craterfree appearance could be the result of a limited data set. But even sparsely cratered Europa expresses craters that would be visible in the limited Io coverage, so clearly the volcanism drives an incredible resurfacing rate. The total absence of any observed crater on Io provides an important minimum value on the resurfacing rate of the body (Johnson and Soderblom 1982). A valuable imaging investigation for any future exploration of Io would include the search for a primary impact crater, and if there is one, an attempt to assess the magnitude of the spawned secondary population.

\section{NEW RESULTS}

In this section, we describe new simulations and some basic theoretical considerations for how the magnitude of the secondary crater population may vary across the solar system.

\section{Relationship Between the Impacting SFD and Relative Abundance of Secondaries}

As described earlier, the amount of mass available for secondary cratering depends on the size of the primary crater, which dictates the total ejected mass, and how much of that mass falls between $v_{\text {min }}$ and $v_{\text {esc }}$. We can generalize to an entire impacting population as follows.

Assume that the mass available for secondary craters from a given primary impact is proportional to the transient primary crater diameter cubed, or $m_{\mathrm{sec}} \propto D^{3}$. For simple craters, the transient crater diameter is close to the final, observed crater diameter. For complex craters, there are differences between the transient crater diameter and final crater diameter. However, this analysis is not sensitive to the particular relationship between transient and final crater diameter. It simply assumes that larger craters generate more ejecta in a fashion that is proportional to the diameter cubed, and that more ejected mass corresponds to more fragments that make secondary craters. While the details of the exact analytical model used to calculate ejecta mass will affect the magnitude difference between crater sizes, our conclusions hold true independent of the exact functional form, as long as the "bigger primary craters create more ejecta than smaller primary craters" assumption is valid.

If the portion of the primary crater population of interest is approximated as a differential power law, with $\frac{\mathrm{dN}}{\mathrm{dD}}=k D^{-b}$ then the total mass available to make secondaries from the primaries is

$$
M_{\mathrm{sec}} \propto \int_{D_{\min }}^{D_{\max }} D^{3} k D^{-b} d D
$$

which evaluates to

$$
\begin{gathered}
M_{\mathrm{sec}} \propto \frac{1}{b-4}\left(D_{\min }^{4-b}-D_{\max }^{4-b}\right) \text { for } b \neq 4, \text { and } \\
M_{\mathrm{sec}} \propto \ln \frac{D_{\max }}{D_{\min }} \text { for } b=4 .
\end{gathered}
$$

All constants were rolled into the proportionality relationship, including the fact that only a fraction of ejected mass is available to make secondary craters (Bierhaus et al. 2012). Table 3 lists the total mass available to make secondaries, modulo the proportionality, for different impactor population indices $b$ (differential power law exponent). For each value of $b$, the primary crater diameter range is from $D_{\min }=1 \mathrm{~km}$ to $D_{\max }=100 \mathrm{~km}$. Recall that as $b$ increases (and thus $-b$ decreases), there are proportionally more and more smaller impactors relative to large impactors. All else being equal (such as generally consistent fragmentation of the bulk mass into discrete ejecta fragments), an impacting population with low $b$ will generate proportionally more secondaries 
Table 3. A comparison of the relative mass available for secondaries for different impacting populations. Because a shallow (smaller b) population has proportionally greater number of larger impacts relative to a steeper (larger b) population, and because the mass available to make secondaries is proportional to the primary crater diameter cubed, a shallow population will have more mass available to make secondaries, and thus generate more secondaries, than a steeper population.

\begin{tabular}{lc}
\hline$b$ (differential power & $\begin{array}{l}M_{\text {sec }} \text { (mass available to } \\
\text { make secondaries) }\end{array}$ \\
\hline law index, or slope) & 4999.5 \\
2.0 & 666.0 \\
3.0 & 99.0 \\
3.5 & 18.0 \\
4.0 & 4.6 \\
4.5 & 1.8 \\
\hline
\end{tabular}

than an impacting population with large $b$, because the low- $b$ population will have more large impacts, which generate the most secondaries. This simple exercise demonstrates that the overall magnitude of the secondary population, relative to the primary population, strongly depends on the SFD of the impacting population itself.

\section{Ejecta Fragment Simulations}

Here we describe simulations that take the integrated masses of the previous discussions, and discretize the total ejected mass into specific ejecta fragment populations for each impact. The methodology is described in Bierhaus, Dones, and Robbins (in preparation), which we summarize below.

1. Assume an impactor flux (details below) with an SFD and impact velocity distribution

2. Use impactor scaling laws (e.g., Housen and Holsapple 2011) to turn the impactors into primary craters

3. Use crater ejecta scaling laws from Housen and Holsapple (2011) and fragment size observations from Singer et al. (2013) to develop fragment ejecta population (SFD and velocity distribution)

i. The fragments are assigned initial positions and velocities based on scaling laws, and their initial conditions are not clustered. Thus, these fragments represent a component of the background secondary population, rather than the "obvious" clustered secondaries often associated with crater rays.

4. Assign a random azimuthal ejection angle $\varphi$ between $0^{\circ} \leq \varphi<360^{\circ}$, where $\varphi$ is the angle between north and the ejection direction. This specifically creates a population of secondaries that simulates the accumulation of background secondaries, i.e., those secondaries that would be included in the measurements after obvious crater clusters are excluded. This assumption underestimates the secondaries present in the measured population, because of the statistical variation in what defines a cluster, i.e., most researchers when avoiding "obvious clusters" discard craters clustered at the $3 \sigma$ level (or more), yet the remaining measurements may include craters clustered at lower confidence levels, see Bierhaus et al. (2005).

5. Integrate fragment trajectories using the WisdomHolman method (Wisdom and Holman 1991) with SWIFT (Levison and Duncan 1994), and generate secondary craters from their impact, recording the impact speed, location, and secondary crater diameter for each fragment

i. We assume $v_{\min }=150 \mathrm{~m} \mathrm{~s}^{-1}$ for icy surfaces, and $v_{\min }=250 \mathrm{~m} \mathrm{~s}^{-1}$ for rocky surfaces

The objective of the simulations is to quantify the contribution of background secondaries to the total measured population of primaries-plus-background secondaries, and compare that with the accumulation of primaries alone. This is a test to determine if the accumulation of primaries-plus-background secondaries preserves an accurate correlation with surface age.

We apply two simplifications to make this simulation numerically tractable on a high-end but single workstation (1) we generate fragments for primary craters large enough that their secondaries would be visible in the images, or primaries $\gtrsim 8 \mathrm{~km}$; and (2) we generate 1000 fragments per primary crater, a significant underestimate. The first simplification is straightforward when interpreting the results, i.e., we have a realistic joint primary-plus-secondary crater population above some size. The second simplification is motivated by the fact that we are simulating the background secondary population only, which is not the total secondary population. Artificially minimizing the secondary population gives us a lower limit to their effect on the local, regional, and global crater SFDs. Any conclusions we draw from these simulations are lower limits because the actual secondary crater population per primary is orders of magnitude larger.

Our simulations include the Moon, a surface that is the basis for many impact-crater based chronologies, and several icy satellites. To develop a primary impactor population, we do the following. In all cases, we assume impacts occur uniformly over the surface of a satellite, i.e., we ignore apex-antapex asymmetries, and take the angle of impact from the normal, $\theta$, to 
follow the standard $\sin (2 \theta)$ distribution (Shoemaker 1962). For icy satellites, we use Zahnle et al. (2003) Case $\mathrm{A}$ to determine the mean rate of impacts, $\lambda$, by "comets" bigger than a minimum diameter $d_{\min }$ on a satellite. We then generate impacts on the satellite for a simulated time $t=4 \mathrm{Ga}$. For instance, for Case A on Tethys, if we assume $d_{\min }=15 \mathrm{~m}$, we have $\lambda=3.95 \times 10^{-6} \mathrm{yr}^{-1}$, so the number of impacts expected is $N=\lambda t=15,800$ (left-hand plot in Fig. 3). We assume impacts are independent of each other, so the time between impacts follows an exponential distribution. We then use the Case A size distributions from Zahnle et al. (2003) to select the size of the impactor. Finally, we choose the impact velocity from a distribution that extends from $\left(2^{\prime \prime}-1\right) v_{K}$ to $\left(2^{\prime \prime}+1\right)$ $v_{\mathrm{K}}$, with median impact velocity $3 " v_{\mathrm{K}}$, where $v_{\mathrm{K}}$ is the orbital speed of the satellite around the planet. This is the appropriate distribution when both the impactor's velocity at "infinity" and the moon's surface escape velocity are much smaller than $v_{\mathrm{K}}$ (Lissauer et al. 1988; Zahnle et al. 2003). For the Moon (right-hand plot in Fig. 3), we assume the impactors are NEAs with the distribution of absolute magnitudes $(H)$ given by Tricarico (2016). We consider impactors with $H<27$, corresponding to diameters $d>14 \mathrm{~m}$ for a geometric albedo of 0.14 . We assume each NEA has an impact probability with the Moon of $7.33 \times 10^{-11} \mathrm{yr}^{-1}$ (Le Feuvre and Wieczorek 2011). For our NEA size distribution, this corresponds to 298 expected impacts by bodies with $d>1 \mathrm{~km}$ in $4 \mathrm{Ga}$. Finally, we assume an impact velocity distribution from Marchi et al. (2009), who took dynamical models of near-Earth objects and Main Belt asteroids by Bottke and colleagues, and then applied a collisional lifetime estimate from Bottke et al. (2005). The velocity distribution thus depends weakly on diameter. Marchi et al. provided velocity distributions for the smallest $(d=0.1 \mathrm{~m})$ and largest $(d=72 \mathrm{~km})$ bodies in their model. The corresponding median impact speeds on the Moon are 15 and $18 \mathrm{~km} \mathrm{~s}^{-1}$. For our simulations, we have used the velocity distributions for the "large" bodies.

Figure 4 plots the results of the simulations for a single primary crater to illustrate the variation in spatial distribution of the resulting secondary crater population for several solar system bodies. Enceladus and the Moon are two endmembers from the simulation that highlight the differences in secondary crater populations. In the case of Enceladus, the spatial distribution is due to the relative proximity of the maximum and minimum speeds at which secondaries can form on the object; this results in a "belt" of secondaries around Enceladus from one impact. The inner limit of the belt is because $v_{\min }=150 \mathrm{~m} \mathrm{~s}^{-1}$ (at $45^{\circ}$ ejection angle) corresponds to a distance of $\sim 300 \mathrm{~km}$ from the ejection location, meaning the "closest" secondaries form far from their parent primary. The outer extent of the belt is the limit to the classic ballistic behavior of the ejecta fragments, or $\sim 200 \mathrm{~m} \mathrm{~s}^{-1}$; fragments moving faster than this velocity are sufficiently perturbed by Saturn's gravity that they often go into orbit around Saturn. The resulting secondary-crater spatial distribution is unlike the "classical" distribution seen for impacts on the terrestrial planets. In contrast, the results for the Moon show a secondary crater population more concentrated around the source primary crater, with a smaller portion of the secondary population achieving global distribution. Thus, in one case-Enceladus-all the secondaries are effectively global in extent, while in another case-the Moon-the majority of the secondaries are local-to-regional in extent.

The other objects in Fig. 4 have results that fall within those two extremes. Tethys and Rhea have distributed but progressively more compact secondary populations because these worlds have larger surface gravities than Enceladus. The other feature of note for these worlds is the spatial relationship between the parent primary and the closest secondaries. Unlike the dense annulus of adjacent secondaries immediately outside the ejecta blanket of a primary crater on, e.g., the Moon or Mars (or even the icy Galilean satellites), there is a gap between the crater center location and the appearance of the closest secondaries. This is because of the $150 \mathrm{~m} \mathrm{~s}^{-1}$ value for $v_{\min }$; a fragment ejected at $150 \mathrm{~m} \mathrm{~s}^{-1}$ and a $45^{\circ}$ angle travels 83,108 , and $179 \mathrm{~km}$ for Rhea, Iapetus, and Tethys, respectively (Bierhaus et al. 2012). The "global scale" of these plots artificially enhances the density of the points of the annulus of secondaries that appear at a range that corresponds to $v_{\text {min }}$; in reality, these points are fairly dispersed. This could explain why secondaries are not obvious on any of the mid-sized Saturnian satellites except Rhea, Saturn's largest mid-sized moon. These results demonstrate that the spatial distribution of secondaries should vary as a result of surface gravity and the ejecta velocity distribution (which is a function of the primary impact speed). The difference in spatial distributions may have consequences for determining the effect of secondaries on a crater-based impact chronology, which we discuss next.

\section{Accumulation of Craters in Time}

Another important capability of this simulation is the ability to track the position and time of each primary and secondary crater formed. By doing so, we can assess local, regional, and global variability of crater density and SFDs as a function of time. 

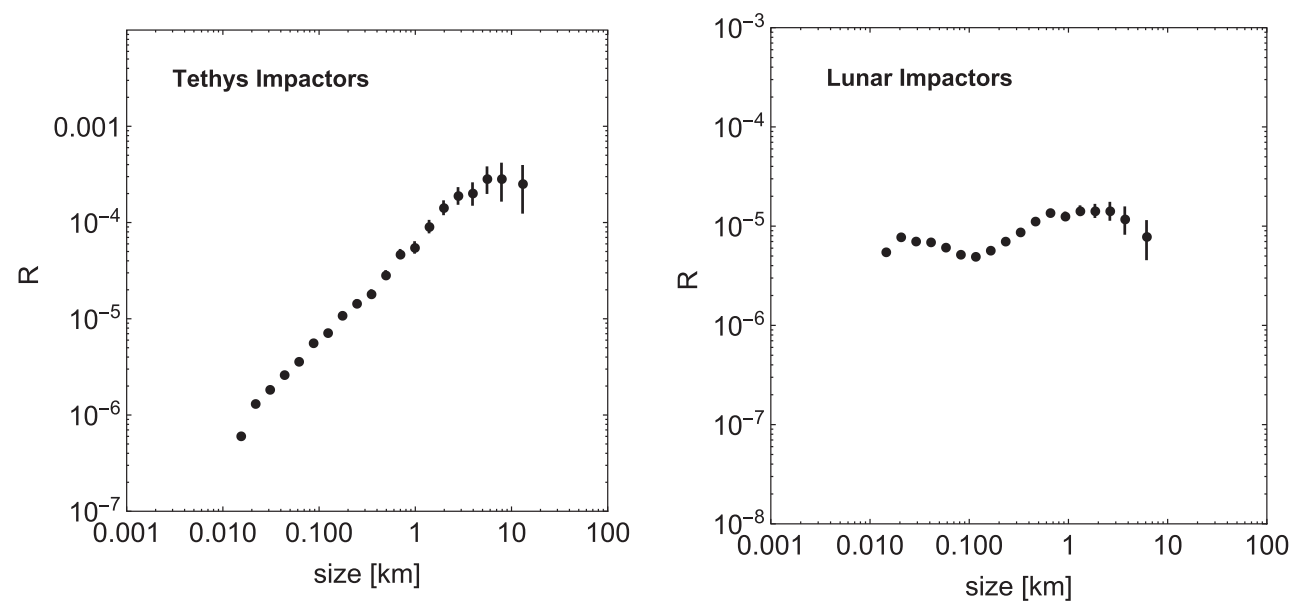

Fig. 3. The impactor populations used for our simulations. For the Saturnian satellites, we used the size-frequency and velocity distributions described in Zahnle et al. (2003); the left plot shows the impactors for Tethys, generated according to Zahnle et al. (2003) Case A. In this simulation, 216 comets with diameters $d>1 \mathrm{~km}$ strike Tethys; the largest has $d=18.5 \mathrm{~km}$. For the lunar impactors (right), we used the NEA SFD described in Tricarico (2016), the impact probability from Le Feuvre and Wieczorek (2011), and velocity distribution from Marchi et al. (2009). The impactors are artificially truncated at $\sim 15 \mathrm{~m}$ diameter in all cases. This truncation causes an edge-effect at the smallest diameter bin such that it plots low. Note the different vertical scales.

To evaluate the accumulation of primary and secondary craters across the surface, we divide each sphere into 102 sectors whose areas differ by no more than a factor of two. In each sector, we monitor the accumulation of primary and secondary craters made by the simulation as distinct populations; the total "observed" population for each quadrant is simply the sum of the two populations. We track the accumulation of craters in each sector, at each time step, which is numerically tractable because we consider primary impactors $>15 \mathrm{~m}$ diameter, and consider only 1000 fragments per primary larger than $\sim 8 \mathrm{~km}$. Because the fragments simulate the "background secondary" population, they are not spatially clustered, other than the inherent falloff in density from the parent primary crater (see Fig. 4).

Figure 5 plots the simulation results for several surfaces. At each time step, the algorithm finds the sectors with the maximum, mean, and minimum crater densities for each of the three crater populationsprimaries only, secondaries only, and combined. There are nine curves in each plot: three curves representing the three populations of primaries only (dashed), spatially random secondaries only (dotted), and the combined population (solid); and for each population, there is the minimum spatial density (light-gray), average spatial density (dark gray), and maximum spatial density (black). Because secondaries dominate the spatial density after some time, the dotted (spatially random secondaries only) and solid (primaries-plussecondaries) curves are very close to one another. We plot the data as time versus spatial density because that corresponds to the age determination process: a researcher measures a crater population in image data, and that spatial density, and corresponding SFD, are used to calculate a surface age. We make several observations regarding the evolution of the spatial densities in time.

First, the difference between the maximum, mean, and minimum values for the primary craters varies by a factor of a few from one another, so even for the same global surface age (or crater retention age, e.g., Hartmann and Neukum 2001), there will be differences across the surface in crater density. Thus, without other complications, a crater-based surface age should be accurate to no better than a factor related to the minimum and maximum possible crater densities at a point in time for a given impact flux. To illustrate this, consider the data for Enceladus, the upper-left plot. At a crater spatial density of 0.001 per $\mathrm{km}^{2}$, the primaries only maximum spatial density (black dashed curve) reaches that value at $\sim 1.6 \times 10^{9} \mathrm{yr}$, whereas the minimum spatial density (light-gray dashed curve) reaches that value at $\sim 2.9 \times 10^{9} \mathrm{yr}$.

Second, the relative rate of accumulation for secondaries compared with primaries is object dependent, consistent with the conclusion derived from the more simple, total mass-based analysis described earlier. A few important examples are as follows.

1. Enceladus - although there are fewer secondary craters forming on the surface per primary impact on Enceladus because of the narrow velocity range in which secondaries form (Bierhaus et al. 2012), the low surface gravity means that the secondaries 

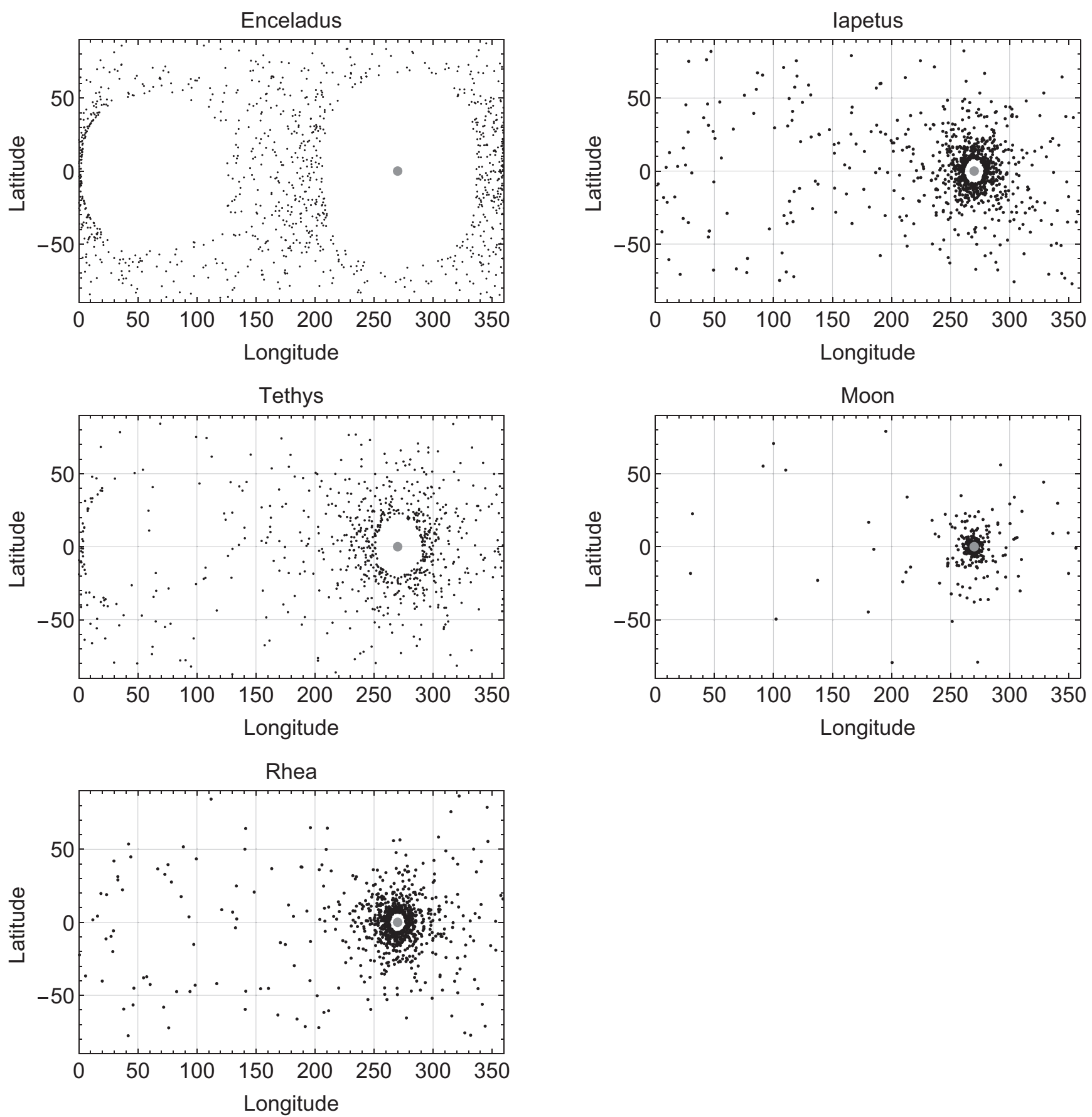

Fig. 4. Plots of the secondary crater population generated by our simulations for different solar system objects. The gray dot marks the location of the primary crater, and the black dots are the locations of secondary impacts. The dots are not representative of size, and only mark locations. The proximity at which secondaries form relative to their parent primary is a function of $v_{\min }$ and surface gravity. We assumed $v_{\min }=150 \mathrm{~m} \mathrm{~s}^{-1}$ for the icy surfaces, and $250 \mathrm{~m} \mathrm{~s}^{-1}$ for the rocky surfaces. Although the actual values may vary, the basic relationship will hold true: as surface gravity decreases (e.g., Enceladus) and/or $v_{\min }$ increases, the minimum range at which secondaries form will increase. This explains why the dense annuli of adjacent secondary craters seen on the Moon, Mars, Mercury, and the large Galilean icy satellites are absent on the mid-sized and small Saturnian satellites.

that do form are more widely distributed across the surface, rather than being concentrated around the parent primary (e.g., Fig. 4). Thus just a few primary impacts create a globally distributed secondary population. While the number of secondaries on Enceladus rapidly exceeds the 

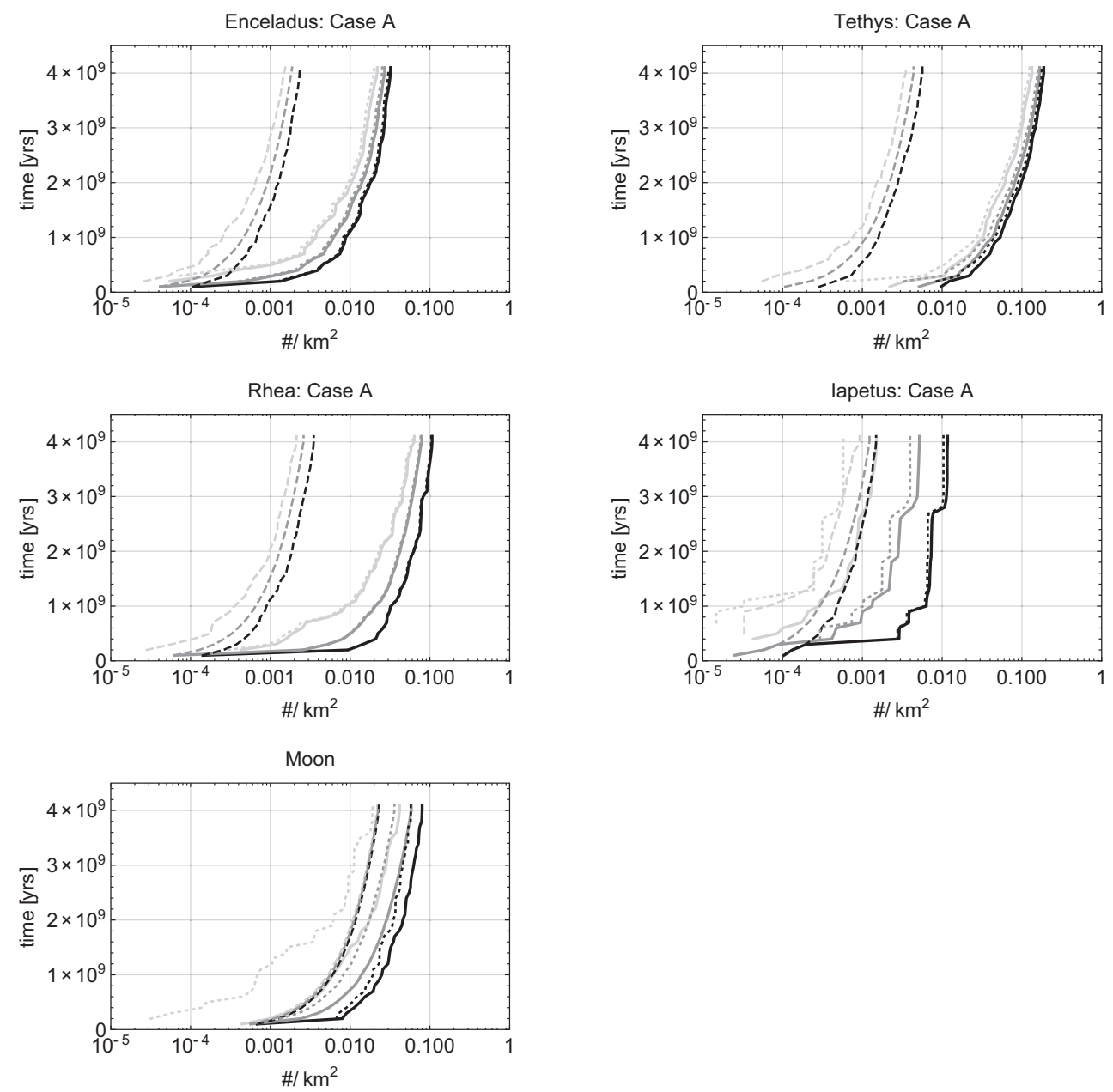

Fig. 5. These plots show the evolution of crater density over time for Earth's Moon, and several Saturnian satellites. The horizontal axis is crater density in $\# / \mathrm{km}^{2}$, and the vertical axis is the time in years. In both cases, there are nine curves, three each for primaries only, secondaries only, and the sum of the two populations. The dashed lines are primaries only, the dotted lines are secondaries only, and the thick solid lines are combined population (primaries plus secondaries). For each group, black line is the maximum spatial density on the surface, the middle dark gray line is the average spatial density, and the light-gray line is the minimum spatial density. See text for discussion.

number of primaries at sizes observable in Cassini data (Table 1), the difference between the maximum and minimum number density of the secondaries, as well as the combined primaries-plus-secondaries population, is generally within a factor of a few.

2. Rhea-although the number density from the accumulation of primary craters never differs by more than a factor of a few between the minimum and maximum curves, there is significant variation between the minimum and maximum number density of the secondary population. This is a result of the increased concentration of a secondary population around its parent primary. The secondary population is more localized than Enceladus, though is still global in extent, see Fig. 4.
3. The Moon-the greater number of impactors (Fig. 3) leads to a faster accumulation of primary craters, the lunar primaries reach higher spatial densities than the Saturnian satellites. The faster impact rate also decreases the difference between the maximum and minimum number densities for primary craters, i.e., the three dashed curves lie close to one another. The minimum secondarycrater number density remains below the primarycrater number densities, though that is certainly an artifact of the small number of simulated secondaries relative to the actual value.

When considering the consequences of these simulations for deriving surface ages based on crater measurements, there are two important behaviors to monitor: the spread in total number density between the 
maximum and minimum values, and the number density evolution over time. The spread in number density defines the variability that is possible for two different surfaces that are the same age. If the spread is small, then the surface age is well constrained. As the range of allowable number densities increases for a given surface age, the uncertainty in age grows as well. The evolution of the number density gives a sense of the "smoothness" of crater populations over time. A curve that increases smoothly over time is more deterministic than one that has large changes in value at discrete events (i.e., due to the creation of a large flux of secondaries). The secondaries only and combined primaries-plus-secondaries data in Fig. 5 are artificially smooth because of the fixed number of secondaries, regardless of the size of the source primary.

There are caveats to consider when evaluating these results. The first relates to the spatial gridding used for the analysis, which is a numerical convenience and provides a straightforward means to compare similarsized areas across the globe, but which is unlike the methodology used to define areas of interest for crater measurement. Often measured areas are selected because they are part of a specific geologic unit, or are defined by the constraints of the available images suitable for crater measurement. Also, the sectors in the simulation are all roughly $\sim 1 \%$ of the surface area, which seems small, but can be large compared with the surface areas commonly measured using very high-resolution image data (e.g., of Mars or the Moon). For example, a $100 \mathrm{~km} \times 100 \mathrm{~km}$ region, imaged at sufficient resolution to sample the small crater populations affected by secondary craters, is a relatively large measurement area, but it is still only $\sim 0.1 \%$ of Rhea's surface area. Thus, the simulation results are a spatial average over scales broader than the areas used for localized studies. Greater variability exists at smaller spatial scales. A second caveat is that the simulations generate only 1000 fragments per primary, which in the case of larger fragments could be too few by orders of magnitude. This causes the secondary spatial-density data to be underestimated, and the evolution of the secondary spatial-density data in time to be artificially smooth. In reality, the maximum spatial density for secondaries would have large jumps in value when the large primary craters form. Finally, these simulations assume a particular impactor flux (Fig. 3); although these impactor populations derive from direct observations as much as possible, they are still models based on an incomplete understanding of both the current impactor fluxes (inner and outer solar system), and the extrapolation of those fluxes back in time.

Ultimately, although these simulations model a subset of the secondary population, they provide a mechanism to understand the specific accumulation of primary, secondary, and combined populations of craters at different spatial scales, from regional to global. The simulations provide increasing distinctions between secondary populations between satellites and planets, and they highlight some of the relationships between primary and secondary populations. Because the simulations use only 1000 fragments per primary, they do not overestimate the influence of spatially random, background secondaries.

\section{Simulated Crater SFDs}

For the simulations described in the previous section, computational limitations, related to the orbital integrations, restricted the number of fragments generated per primary. This limitation prevents a complete assessment of the relative SFDs of primary and secondary craters. Here we describe a variation on the simulations in the previous section that improves the assessment of primary, secondary, and combined SFD evolutions.

By focusing exclusively on the SFD aspect of the simulation, we avoid the need to integrate the fragments' positions and velocities. We simply create the fragments as before, assume the fragment impact speed is the same as the ejection speed, and use a scaling law to generate a secondary crater from the fragment.

As before, we assume ejected mass is proportional to the primary crater diameter cubed, and that this relationship is self-similar for any two diameter ranges with the same relative factor between minimum and maximum diameter. For example, the results described below are for primary craters between roughly $50 \mathrm{~m}$ and almost $6 \mathrm{~km}$ in diameter, or a factor of $\sim 140 \times$ in primary crater diameter. As long as that same diameter range of $140 \times$ is maintained, the relative proportion of primaries to secondaries should remain the same, even if the absolute values of the minimum and maximum diameters change.

The simulation outline for this analysis is as follows.

1. Generate a primary impactor population according to a given differential slope, and a fixed total number of impactors.

2. From those primary impactors, and a distribution of impact velocities (speed and impact angles) appropriate for each body, generate primary craters using Housen and Holsapple (2011).

3. For each primary crater, generate secondary fragments. Generate fragment diameter and velocities according to scaling relationships defined in Housen and Holsapple (2011), with the number of largest secondaries per primary based on the data from Singer et al. (2013). 
4. Accumulate a running sum of ejected mass per primary as each fragment is generated, and stop the fragment generation once the running sum reaches the total mass available to make secondaries for that primary, or when there are $10^{5}$ fragments for that primary, whichever comes first.

5. Use the strength scaling relationship in Housen and Holsapple (2011) to calculate the secondary craters made from the ejected fragments given their diameter, assumed density, and impact velocity.

Limiting the maximum number of fragments per primary to $10^{5}$ is a practical consideration because each of the larger primary craters could generate $\gg 10^{6}$ fragments; when multiplied by the number of larger craters, the calculation becomes computationally prohibitive on available desktop workstations. To compensate for this limitation, in these simulations we focus on smaller diameter primary craters with lower overall ejected mass, and for which $10^{5}$ fragments represent either all or the majority of mass available to make secondaries. And as described above, the relative proportion of primaries to secondaries should remain the same for a given primary-crater diameter range, even if the minimum/maximum values of that diameter range shift to larger (or smaller) values. These simulations improve the completeness of the secondarycrater population by a factor of $100 \times$ relative to the simulations with $10^{3}$ fragments per primary. We address the completeness of the generated fragment populations below.

We use three different model impactor populations, with differential slopes $-2,-3$, and -4 . A -2 differential slope is consistent with the primary craters seen on young terrains of the icy satellites, especially Europa and Enceladus, for which there is a clear primary population (and which is supported by the New Horizons observations of Pluto and Charon, see Robbins et al. [2017] and Singer et al. [2016]). A -4 differential slope bounds the maximum expected SFD for small primary craters on the Moon. (The Neukum et al. [2001] lunar production function assumes minimal contribution from secondaries at diameters $<1 \mathrm{~km}$, and their polynomial description of the SFD at these diameters varies between differential slopes of -3 to -3.8.) We use Tethys as a reference target body (to establish impact speeds, surface gravity, escape speed, and target strength). The impactors are between 1 and $250 \mathrm{~m}$ in diameter, resulting in primary craters roughly between $40 \mathrm{~m}$ and $\sim 6 \mathrm{~km}$ diameter. To assess the effect of primary-crater density, and the validity of a given number of impactors to capture the relative importance of primary and secondary craters, we ran two cases for each differential population, one with $10^{3}$ impactors, and one with $10^{4}$ impactors.
Figure 6 plots the results of the simulations. Panels (a), (b), and (c) show the $10^{3}$ impactors (gray data) and $10^{4}$ impactors (black data) for the $-2,-3$, and -4 differential slope primary impactors. The filled black circles are the resulting primary craters, while the open circles are the secondary craters made by the primaries. The $10^{4}$ impactor simulations, while more completely sampling the impactor diameters, do not substantively change the outcome from the $10^{3}$ impactor simulations. These results illustrate some key relationships between the slope of the impacting population, the primary craters made by those impactors, and the resulting secondary craters.

First, these simulations reinforce a conclusion from the simple analytical exercise described earlier, namely that a shallow-sloped primary population will generate more secondaries compared with a steeper primary population over the same diameter range, assuming comparable impact frequencies at a given diameter range. This is a more detailed and explicit illustration of the calculation summarized in Table 3. Second, it is hard to shift the "crossover" diameter to larger sizes as a crater population matures for a population that follows a single power law exponent, where the crossover diameter is the crater size at which secondaries become roughly equivalent to primaries in number. That is because there are only a few "large" secondaries (near $0.05 D_{\mathrm{p}}$, where $D_{\mathrm{p}}$ is the primarycrater diameter) per primary crater, and even for shallow-sloped primary populations, there will always be more primaries at that size than secondaries. As a surface accumulates more and larger primaries with secondaries around $0.05 D_{\mathrm{p}}$, the growth of secondaries at that size will always be outpaced by primaries at that size. This conclusion may not hold true for more complex impactor SFDs, which are not wellapproximated by a single power law exponent. Third, the secondary-crater SFD is a function of the primarycrater SFD. We discuss this further, using Fig. 7 as the central illustration of this point.

Figure 7 plots only the secondary crater populations for each of the three impacting populations $\left(-2,-3\right.$, and -4 differential), as well as both the $10^{3}$ impactor (gray data) and the $10^{4}$ impactor (black data) versions of those populations. For each set of gray and black data, the lowest curve is for the secondaries from the -4 differential primary population, and the middle and top curves are from the -3 and -2 differential primary populations, respectively. The data show that the steeper the primary SFD, the steeper the secondary SFD. The secondaries from the -2 differential primary population display a roughly -4 differential slope, while the secondaries from the -4 differential primary population display a nearly -5 differential slope. As 

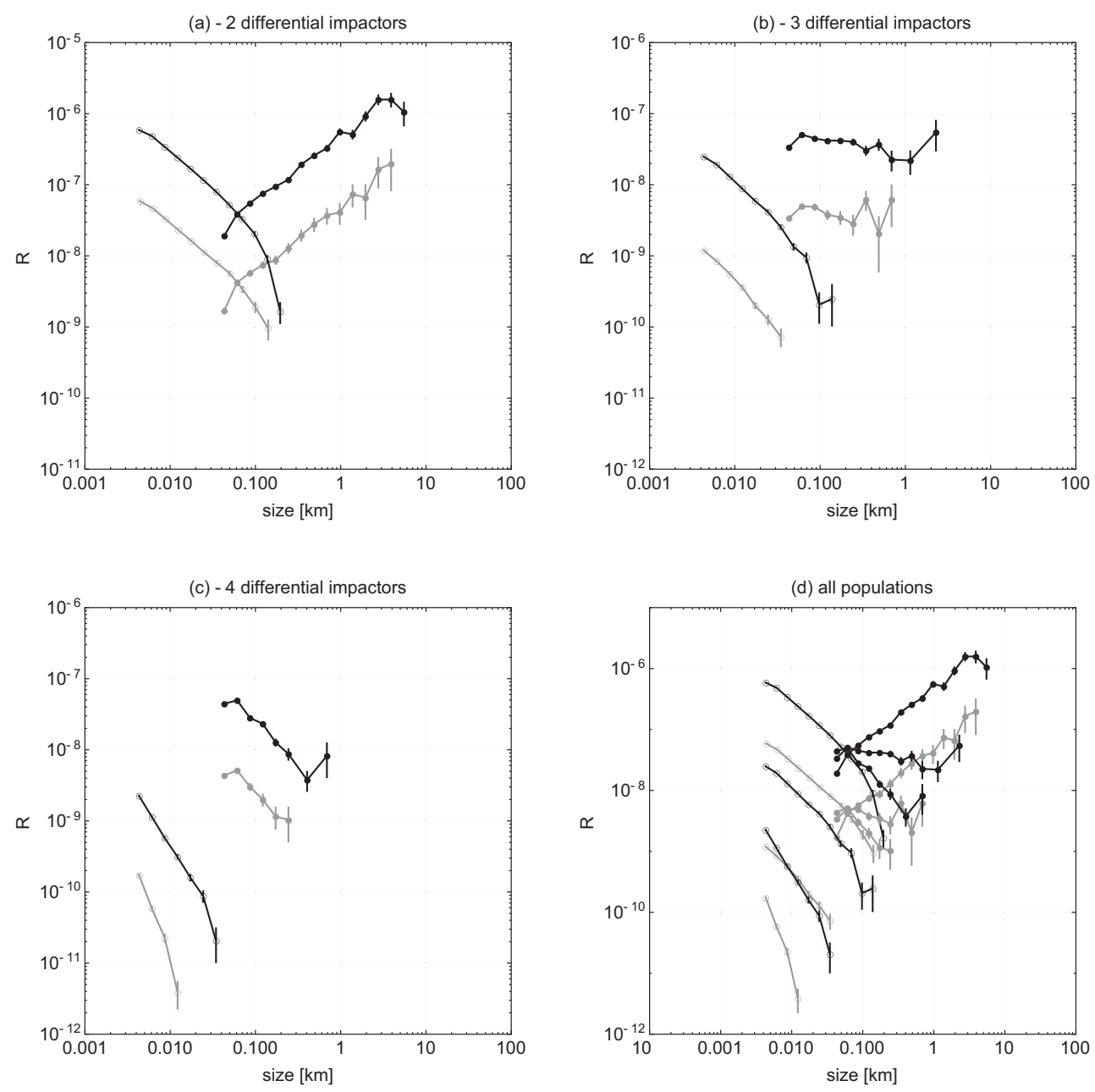

Fig. 6. Simulated primary and secondary crater populations for three differential slopes, $-2,-3$, and -4 . Panels (a), (b), and (c) have four data sets: the gray data are for $10^{3}$ primary impactors, the black data are for $10^{4}$ impactors; the closed circles are the primary craters, the open circles are the secondaries made by the primary craters. Panel (d) is a combination of the other panels to facilitate comparison of all results. Note the change in scales between the panels. In each primary population, small number statistics introduce departures from the power law at the large sizes, and the artificial lower limit to the impactor diameters causes a dip in the smallest diameter bin.

introduced in earlier sections, this behavior results from the varying mass available to make secondaries. The greater proportion of larger primary craters for a shallow impacting SFD results in larger secondaries (made by the larger primaries). As the impacting SFD steepens, and the primary craters are biased toward smaller diameters, there is less mass available to make secondaries, and that mass goes into making smaller fragments from the smaller primaries. The relative absence of larger fragments, and thus the relative absence of larger secondaries, steepen the secondarycrater SFD.

The caveats to this simulation are that no impacting population follows a single power law relationship, the diameter range of primaries is limited relative to a typical planetary surface, and the number of fragments is limited to $10^{5}$ per primary. A realistic impacting population changes slope over its full diameter range, and larger craters can have orders of magnitude more secondaries than the $10^{5}$ limit. Nevertheless, the general principles of this simulation should remain true for the segments of real populations that can be approximated as power laws. For a given diameter range that can be approximated as a power law, a shallower slope results in a greater relative abundance of secondary craters because there is more mass available to make secondaries from a shallower impacting population when compared to a steeper impacting population.

Figure 8 is a histogram that plots the fraction of $M_{\text {sec }}$ (the total mass available to make secondaries) modeled for the -2 differential, $10^{4}$ impacting population, which has the most mass for secondaries 


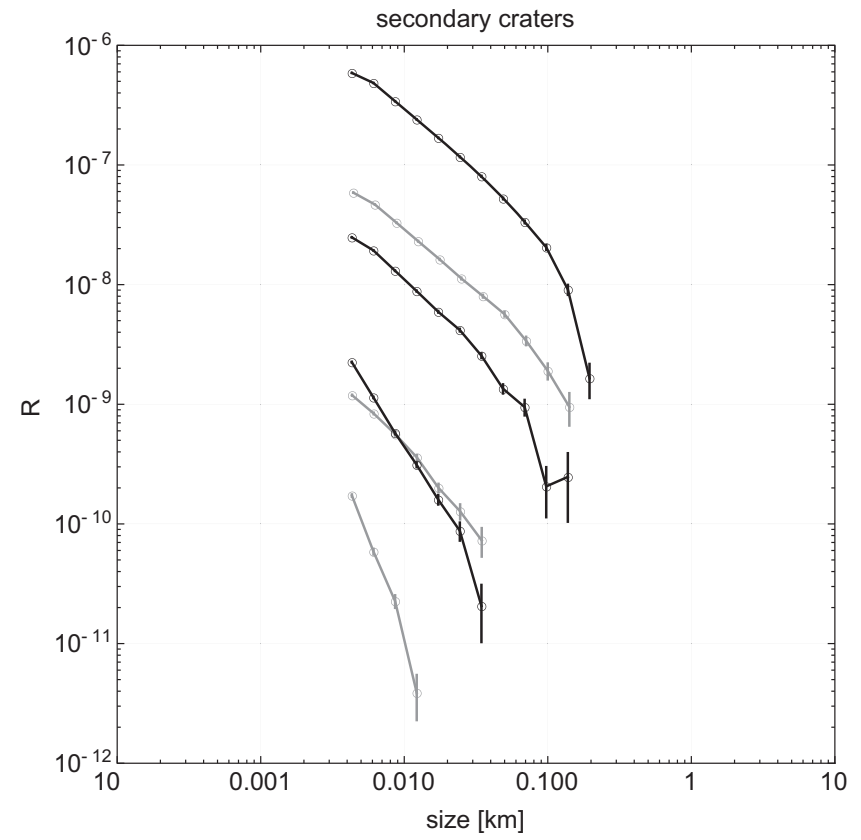

Fig. 7. The secondaries only from the simulations shown in Fig. 6. The gray data are secondaries from the $10^{3}$ impactor population, and the black data are the secondaries from the $10^{4}$ impactor population. For each color, from bottom curve to top curve, the secondaries are for the -4 differential, -3 differential, and -2 differential primary population, respectively. These data show that the secondary SFD steepens as the primary crater SFD steepens.

out of the six simulations. The secondary population is completely modeled, or nearly completely modeled, within the $10^{5}$ fragment limit for the majority of the craters. (This was by design - the reason for simulating small primary craters is because they are most likely to have secondary populations that do not exceed $10^{5}$ members.) If the self-similarity for the relationship between primary-crater diameter range and ejected mass holds true in a relative sense for a fixed diameter range, then the relative secondary populations should be the same for larger primary crater diameters.

\section{CONSEQUENCES FOR CRATER SFDS AND AGE DETERMINATION}

Here we apply the review of earlier work, and the new results in this paper, to refine our understanding of the evolution of crater SFDs, and the consequences for surface age estimation, across the solar system. Previous analyses typically considered endmember solutions for the effect of secondaries on crater SFDs and age determination, either (1) secondaries were inconsequential for age estimates because they are an unimportant fraction of the overall population (e.g.,

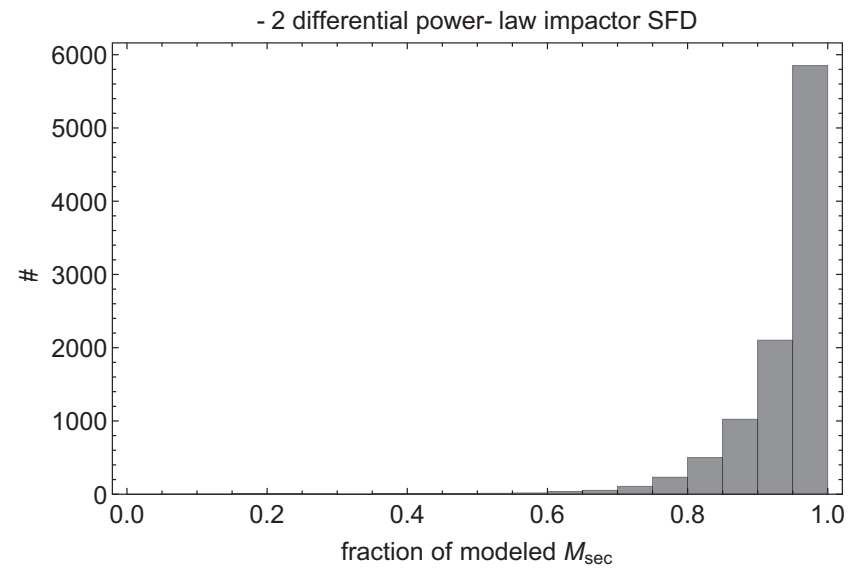

Fig. 8. A histogram for the fraction of modeled $M_{\mathrm{sec}}$ for the -2 differential, $10^{4}$ impactor population. $M_{\mathrm{sec}}=1$ corresponds to a fully modeled secondary population, $M_{\mathrm{sec}}=0.5$ means that only $50 \%$ of the secondary population was modeled. This histogram is from the most incomplete modeling, namely the case with the most large craters, and thus the most craters whose fragment population exceeds $10^{5}$ fragments. Even in this case, most of the craters have complete, or nearly complete, modeled secondary populations.

Neukum et al. 2001) or even if they exist in large numbers (e.g., Hartmann 2007), or (2) secondaries impose significant errors in age estimates, and measuring crater SFDs below some diameter has too much uncertainty to reliably determine an age (Bierhaus et al. 2005; McEwen et al. 2005). Based on results discussed in the previous section, there is probably a continuum between those two endmember conclusions.

Before discussing the effect of secondaries in detail, first we address primary craters. The thin, solid lines in Fig. 5 plot the accumulation of primary craters as a function of time. Even though the entire surface is the same age at each time step, there is a range of crater densities at different locations simply due to the inherent variability stemming from the statistical accumulation of primaries. Furthermore, the spread between the minimum, mean, and maximum primarycrater densities changes as a function of time. That means that a crater-based chronology, even in idealized form, can constrain the age to an accuracy no better than that allowed by the spread in primary crater densities that are possible at a given surface age, assuming that surface age is known.

As for secondaries, the central question for chronology is whether or not, or to what degree, secondaries invalidate use of small crater populations for age determination. A different way of asking the same question is whether the accumulation of secondaries with time preserves the deterministic relationship between measured crater density and 
surface age. The answer to these questions depends on the relative number of primaries and secondaries that accumulate, and their spatial distribution. The accuracy of the deterministic relationship between crater density and surface age is shown in Fig. 5. The greater the discrepancy between the minimum and maximum crater density curves at a given point in time, the less accurate a crater-based chronology (using crater diameters populated by secondaries) will be. Conversely, as the discrepancy between the minimum and maximum crater density decreases, the accuracy of a crater-based chronology improves.

Of course, the crater-based chronology technique measures crater density and SFD, and uses these to infer an age. Our simulations that model the accumulation of primaries and spatially random secondaries across a surface provide an initial understanding of the evolution of the total crater density (primaries plus secondaries) compared with primaries alone, but because we do not model the complete secondary population, we cannot assess the SFD. Examining spatial density alone, Fig. 5 shows that the combined density of primaries-plus-secondaries evolves differently between the objects, and one cannot reach a universal conclusion that applies to all objects; rather, the outcome depends on the specifics of the target.

In the case of our second simulation type that specifically considers the relationship between primary SFD and the resulting secondary SFD, we see that as the impacting SFD steepens, so too does the resulting secondary SFD, although the steepening of the secondary SFD occurs more slowly in response to a steepening primary SFD. The -2 differential primary population produces an approximately -4 differential secondary population, compared with a -4 differential impacting population that produces an approximately -5 differential secondary population. In addition, a shallow-sloped impactor population produces proportionally more secondaries that a steep-sloped impactor population. The combination of these effects - a greater contrast in SFDs between primaries and secondaries, plus proportionally more secondariessuggests surface ages derived from a shallow-sloped production function may be more affected by secondaries.

The differences found in the results presented here further enhance the variability in secondary populations described in Bierhaus et al. (2012), who found that the mass available for secondaries (a proxy for the total number of secondaries) depends on impact speed, surface gravity, target $v_{\mathrm{esc}}$, and target $v_{\min }$. The spatial distribution of secondaries depends on surface gravity and the velocity distribution of the ejecta. Because these factors (impact speeds, surface gravity, target properties, $v_{\text {esc }}, v_{\min }$, and impactor SFD) vary across the solar system, one would expect different secondary populations across the solar system, rather than a monolithic outcome that applies to all cratered surfaces.

These results illustrate the importance of understanding the full suite of factors that contribute to the evolution of a crater SFD. The details of different impacting populations and impact speeds, surface properties, and sizes lead to differences in the primary and secondary crater SFDs between targets, as well as variation in time on the same target. The differences can lead to strongly divergent crater densities on the same surface. Understanding the effect of secondaries on a crater-based chronology is not a function of a single parameter, but rather a combination of several parameters.

Because our current work demonstrates the sensitivity of particular parameters to a given outcome, we do not extrapolate our accuracy estimates to surfaces not yet simulated-i.e., Mercury, Mars, and other icy satellites. Because of the long-standing similarities in the overall crater populations observed on the Moon and Mars, it is certainly reasonable to assume that their outcomes would be comparable, but the Martian atmosphere and active surface processes make a direct comparison complicated (see also Williams et al. [2018]). Observations of Mercury and Europa (Bierhaus et al. 2005) provide strong evidence for a secondary-dominated small crater population for these surfaces.

We reiterate there are some caveats to consider relative to our results. In our simulations that explore the relationship between primary SFD and secondary SFD, we use single-slope primary crater production functions, known to not represent the SFD of the full diameter range of crater populations observed on the surfaces across the solar system, where larger craters have a varying slope. The simulations that track the accumulation of secondaries in time and space are limited to 1000 fragments per primary, and generate secondaries only for primaries above a certain size. As a result, our simulated secondary populations are an underestimate of the true population. Also, we spatially bin the crater populations in a spherical grid of nearly equal-sized sectors. Though each sector is only $\sim 1 \%$ of the total surface area, a sector nevertheless represents significantly more area than some studies that use very high-resolution images to measure the small-crater population. Finer scale spatial binning results in greater variability between bins, which would degrade the age-accuracy estimates we quote above. 


\section{CONCLUSIONS AND SUMMARY}

In this paper, we have summarized current observations of secondary craters across the solar system, from Mercury to Pluto, and provide new work that explores the formation and evolution of primary and secondary crater populations. The available data for observing and characterizing secondary-crater populations continue to expand in the number of targets, areal coverage, and spatial resolution, enabling important advances in our understanding of this fundamental process for midsized and larger bodies. We have unprecedented opportunity to identify the secondary crater population from individual primaries, as well as the ensemble secondary population on a planetary surface. However, the community still lacks consensus in regard to the effect, or not, of secondary craters on crater-based chronologies, with little convergence even after a decade since the last review, and $50 \mathrm{yr}$ since the first peer-reviewed papers on the topic.

Our new results in the previous two sections attempt to resolve the ongoing competing viewpoints in the context of recent observations from across the solar system. The most important general conclusion from recent work, as well as a broad conclusion from new results in this paper, is that secondary-crater populations are multifaceted, and while some general aspects remain consistent between cratered surfaces, each target body will express a unique secondary crater population that depends on several factors. While broad conclusions regarding secondary-crater populations are possible between worlds that share similar factors, the secondary-crater populations will vary between worlds and satellites. We summarize the important similarities and differences below.

Secondary-crater similarities:

1. Secondary-crater SFDs are variable, and are generally steep, meaning $b>3$, and often exceed $b>4$ (where $b$ is the differential power law exponent).

i. The maximum diameter secondary for a given primary is typically between $0.05 D_{\mathrm{p}}$ and $0.1 D_{\mathrm{p}}$, where $D_{\mathrm{p}}$ is the parent primary diameter.

ii. While some secondaries reach this size, the vast majority of secondaries from a given primary impact are smaller.

iii. The presence of larger secondaries on Mercury (high mean impactor speed), in conjunction with the absence of secondaries and Pluto and Charon (low mean impactor speed), suggests that impactor velocity may play a role in fragment ejection.

2. The physics of the crater excavation process results in an inverse mass-velocity relationship; i.e., less mass is ejected at higher velocities, more mass is ejected at lower velocities.

3. The largest ejected fragments are the slowest moving.

4. There is a minimum speed required for bolides to form secondary craters, $v_{\min }$, and that minimum speed likely changes between target surfaces.

Secondary-crater differences:

1. Because the secondary SFD is a function of the primary SFD, different impactor SFDs will generate different secondary crater SFDs.

i. The steeper the primary SFD, the steeper the secondary SFD, and vice versa.

ii. After New Horizons observations of small craters on Pluto and Charon, it is clear that the comet population SFD (derived from small KBOs) differs from the asteroid population SFD. Because comets are the predominant impactor on the icy satellites of the giant planets, and asteroids are the predominant impactor on terrestrial planets, we conclude that both the primary-and thus the secondary-crater populations between the terrestrial planets and icy outer-planet surfaces should be different, even ignoring other factors.

2. The minimum distance from the primary at which secondaries appear depends on surface gravity and $v_{\min }$.

3. The spatial distribution of secondaries depends on surface gravity, which varies between objects.

4. Target properties will affect the production and distribution of secondaries. Though not directly discussed in this paper, target properties affect the size of a primary crater, and thus the amount of mass available to make secondaries. Also, because many secondaries form in the strength regime, target strength affects the resulting secondary SFD. And while not well quantified, $v_{\min }$ should vary between target strengths, with stronger targets causing increased $v_{\min }$.

A "first-order" treatment of secondary-crater populations, using the mass available to make secondaries as a proxy (Bierhaus and Dones 2012; Bierhaus et al. 2012), generated results consistent with several observations available at the time, specifically that Europa should have the most significant secondary population of any giant-planet icy satellite, Rhea should have the most secondaries for the midsized Saturnian satellites, and Mercury should have significantly more secondaries than the Moon or Mars. We advance those conclusions here via simulations that model the accumulation of primary and secondary crater populations as a function of time. The simulations use: current best estimates of the impactor populations to generate the primary 
craters; crater ejecta scaling laws to generate realistic ejecta fragments that comply with known massvelocity relationships; and SWIFT (a well-established numerical integrator, Levison and Duncan 1994) to compute the trajectories of ejecta fragments, tracking their impact locations and speeds. The results of these simulations further emphasize the variability of secondary-crater populations.

Because the contributing factors to secondary craters vary across the solar system, secondary-crater populations do not have a consistent accumulation in time, and therefore affect age estimate accuracies to varying degrees. Secondaries accumulate at different rates between surfaces, and the relative proportion (and thus age accuracy) of primaries and secondaries changes in time for the same surface. It is crucial to account for the different factors that dictate the number and extent of secondary craters, for the factors can combine in ways that result in different crater-derived age uncertainties between planetary surfaces.

Acknowledgments-E. B. B. acknowledges NASA CDAPS grant NNX14AD55G. S. J. R. acknowledges three funding sources: NASA CDAPS grant NNX14AD55G, NASA SSW grant NNX15AH97G, and NASA PGG grant NNX14AP51G. M. R. K. was supported by NASA SSERVI. J.-P. W. was supported by a NASA Mars Data Analysis Program Grant No. NNX14AM12G.

\section{Editorial Handling-Dr. Gordon Osinski}

\section{REFERENCES}

Alpert A. J. and Melosh H. J. 1999. Fragment sizes of high speed ejecta from a large impact on Europa (abstract). 30th Lunar and Planetary Science Conference. p. 1881.

Alvarellos J. L., Zahnle K. J., Dobrovolskis A. R., and Hamill P. 2005. Fates of satellite ejecta in the Saturn system. Icarus 178:104-123.

Alvarellos J. L., Zahnle K. J., Dobrovolskis A. R., and Hamill P. 2008. Transfer of mass from Io to Europa and beyond due to cometary impacts. Icarus 194:636-646.

Anderson L. B., Schultz P. H., and Heineck J. T. 2003. Asymmetry of ejecta flow during oblique impacts using three-dimensional particle image velocimetry. Journal of Geophysical Research: Planets 108(E8). https://doi.org/10. 1029/2003je002075.

Artemieva N. and Ivanov B. 2004. Launch of Martian meteorites in oblique impacts. Icarus 171:84-101.

Artemieva N. A. and Zanetti M. 2016. Modeling small impact craters on ejecta blankets: Self-secondaries versus unrecognizable primaries (abstract \#2143). 47th Lunar and Planetary Science Conference. CD-ROM.

Baker M. H. and Head J. W. 2015. Extensive Middle Amazonian mantling of debris aprons and plains in Deuteronilus Mesae, Mars: Implications for the record of mid-latitude glaciation. Icarus 260:269-288.
Bart G. D. and Melosh H. J. 2010a. Impact into lunar regolith inhibits high-velocity ejection of large blocks. Journal of Geophysical Research: Planets 115:E08004.

Bart G. D. and Melosh H. J. 2010b. Distributions of boulders ejected from lunar craters. Icarus 209:337-357.

Bierhaus E. B. and Dones L. 2012. Cratering by impact ejecta, from Mercury to the asteroids (abstract \#1659). 43rd Lunar and Planetary Science Conference. CD-ROM.

Bierhaus E. B. and Dones L. 2015. Craters and ejecta on Pluto and Charon: Anticipated results from the New Horizons flyby. Icarus 246:165-182.

Bierhaus E. B. and Schenk P. M. 2010. Constraints on Europa's surface properties from primary and secondary crater morphology. Journal of Geophysical Research: Planets 115:12,004.

Bierhaus E. B., Chapman C. R., Merline W. J., Brooks S. M., and Asphaug E. 2001. Pwyll secondaries and other small craters on Europa. Icarus 153:264-276.

Bierhaus E. B., Chapman C. R., and Merline W. J. 2005. Secondary craters on Europa and implications for cratered surfaces. Nature 437:1125-1127.

Bierhaus E. B., Dones L., Alvarellos J. L., and Zahnle K. 2012. The role of ejecta in the small crater populations on the mid-sized Saturnian satellites. Icarus 218:602-621.

Bottke W. F., Durda D. D., Nesvorný D., Jedicke R., Morbidelli A., Vokrouhlický D., and Levison H. F. 2005. Linking the collisional history of the main asteroid belt to its dynamical excitation and depletion. Icarus 179:63-94. Erratum in Icarus 183:235-236.

Bottke W. F. Jr., Vokrouhlický D., Rubincam D. P., and Nesvorný D. 2006. The Yarkovsky and YORP effects: Implications for asteroid dynamics. Annual Review of Earth and Planetary Sciences 34:157-191.

Bottke W. F., Vokrouhlický D., and Nesvorný D. 2007. An asteroid breakup $160 \mathrm{Myr}$ ago as the probable source of the K/T impactor. Nature 449:48-53.

Brown P., Spalding R. E., ReVelle D. O., Tagliaferri E., and Worden S. P. 2002. The flux of small near-Earth objects colliding with the Earth. Nature 420:294-296.

Brown P. G., Assink J. D., Astiz L., Blaauw R., Boslough M. B., Borovička J., Brachet N., Brown D., Campbell-Brown M., Ceranna L., Cooke W., de Groot-Hedlin C., Drob D. P., Edwards W., Evers L. G., Garces M., Gill J., Hedlin M., Kingery A., Laske G., Le Pichon A., Mialle P., Moser D. E., Saffer A., Silber E., Smets P., Spalding R. E., Spurný P., Tagliaferri E., Uren D., Weryk R. J., Whitaker R., and Krzeminski Z. 2013. A 500-kiloton airburst over Chelyabinsk and an enhanced hazard from small impactors. Nature 503:238-241.

Carr M. H. 2006. The surface of Mars. Cambridge, UK: Cambridge University Press.

Cellino A., Michel P., Tanga P., Zappalà V., Paolicchi P., and Dell'Oro A. 1999. The velocity-size relationship for members of asteroid families and implications for the physics of catastrophic collisions. Icarus 141:79-95.

Chapman C. R. and McKinnon W. B. 1986. Cratering of planetary satellites. In Satellites, edited by Burns J. A. and Matthews M. S. Tucson, Arizona: The University of Arizona Press. pp. 492-580.

Chapman C. R., Veverka J., Belton M. J. S., Neukum G., and Morrison D. 1996. Cratering on Gaspra. Icarus 120:231-245.

Cintala M. J., Berthoud L., and Hörz F. 1999. Ejectionvelocity distributions from impacts into coarse-grained sand. Meteoritics \& Planetary Science 34:605-623. 
Crater Analysis Techniques Working Group. 1979. Standard analysis techniques for presentation and analysis of crater size-frequency data. Icarus 37:467-474.

Culler T. S., Becker T. A., Muller R. A., and Renne P. R. 2000. Lunar impact history from 40Ar/39Ar dating of glass spherules. Science 287:1785-1788.

Daubar I. J., McEwen A. S., Byrne S., Kennedy M. R., and Ivanov B. 2013. The current Martian cratering rate. Icarus 225:506-516.

Daubar I. J., McEwen A. S., Byrne S., Kreslavsky M., Saper L., and Kennedy M. R. 2014. New dated impacts on Mars and the current cratering rate. LPI Contribution 1800. Tucson, Arizona: Lunar and Planetary Science Institute.

Daubar I. J., McEwen A. S., Byrne S., Kreslavsky M., Saper L., Kennedy M. R., and Golombek M. P. 2015. Current state of knowledge of modern Martian cratering. Workshop on Issues in Crater Studies and the Dating of Planetary Surfaces. LPI Contribution 1841. Houston, Texas: Lunar and Planetary Institute. 9007 p.

De Pablo M. A., Michael G. G., and Centeno J. D. 2013. Age and evolution of the lower NW flank of the Hecates Tholus volcano, Mars, based on crater size-frequency distribution on CTX images. Icarus 226:455-469.

Dundas C. M. and McEwen A. S. 2007. Rays and secondary craters of Tycho. Icarus 186:31-40.

Dundas C. M., Keszthelyi L. P., Bray V. J., and McEwen A. S. 2010. Role of material properties in the cratering record of young platy-ridged lava on Mars. Geophysical Research Letters 37:L12203.

Durda D. D., Chapman C. R., Merline W. J., and Enke B. L. 2012. Detecting crater ejecta-blanket boundaries and constraining source crater regions for boulder tracks and elongated secondary craters on Eros. Meteoritics \& Planetary Science 47:1087-1097.

Farinella P., Vokrouhlický D., and Hartmann W. K. 1998. Meteorite delivery via Yarkovsky orbital drift. Icarus 132:378-387.

Goudge T. A., Mustard J. F., Head J. W., and Fasset C. I. 2012. Constraints on the history of open-basin lakes on Mars from the composition and timing of volcanic resurfacing. Journal of Geophysical Research 117. https:// doi.org/10.1029/2012JE004115.

Grady D. E. 2008. Fragment size distributions from the dynamic fragmentation of brittle solids. International Journal of Impact Engineering 35:1557-1562.

Grady D. E. and Kipp M. E. 1985. Geometric statistics and dynamic fragmentation. Journal of Applied Physics 58:1210-1222.

Grady D. E. and Kipp M. E. 1987. Dynamic rock fragmentation. In Fracture mechanics of rock, edited by Atkinson B. K. San Diego, California: Academic. pp. 429-475.

Granvik M., Morbidelli A., Jedicke R., Bolin B., Bottke W. F., Beshore E., Vokrouhlický D., Delbo M., and Michel P. 2016. Super-catastrophic disruption of asteroids at small perihelion distances. Nature 530:303-306.

Greenstreet S., Gladman B., and McKinnon W. B. 2015. Impact and cratering rates onto Pluto. Icarus 258:267-288.

Hartmann W. K. 1984. Does crater 'saturation equilibrium' occur in the solar system? Icarus 60:56-74.

Hartmann W. K. 1995. Planetary cratering I: Lunar highlands and tests of hypotheses on crater populations. Meteoritics \& Planetary Science 30:451-467.

Hartmann W. K. 2005. Martian cratering VIII: Isochron refinement and the chronology of Mars. Icarus 174:294-320.
Hartmann W. K. 2007. Martian cratering 9: Toward resolution of the controversy about small craters. Icarus 189:274-278.

Hartmann W. K. and Barlow N. G. 2006. Nature of the Martian uplands: Effect on Martian meteorite age distribution and secondary cratering. Meteoritics \& Planetary Science 41:1453-1467.

Hartmann W. K. and Daubar I. J. 2017. Martian cratering 11. Utilizing decameter scale crater populations to study Martian history. Meteoritics \& Planetary Science 52:493510 .

Hartmann W. K. and Gaskell R. W. 1997. Planetary cratering 2: Studies of saturation equilibrium. Meteoritics \& Planetary Science 32:109-121.

Hartmann W. K. and Neukum G. 2001. Cratering chronology and the evolution of Mars. Space Science Reviews 96:165194.

Hartmann W. K., Quantin C., and Mangold N. 2007. Possible long-term decline in impact rates. 2. Lunar impact-melt data regarding impact history. Icarus 186:11-23.

Head J. N., Melosh H. J., and Ivanov B. A. 2002. Martian meteorite launch: High-speed ejecta from small craters. Science 298:1752-1756.

Hirata N. and Nakamura A. M. 2006. Secondary craters of Tycho: Size-frequency distributions and estimated fragment size-velocity relationships. Journal of Geophysical Research (Planets) 111. https://doi.org/10.1029/2005je 002484.

Hoogenboom T., Johnson K. E., and Schenk P. M. 2015. Contribution of secondary craters on the Icy Satellites: Results from Ganymede and Rhea (abstract \#2530). 46th Lunar and Planetary Science Conference. CD-ROM.

Housen K. R. and Holsapple K. A. 2011. Ejecta from impact craters. Icarus 211:856-875.

Hynek B. M., Robbins S. J., Mueller K., Gererpline J., Osterloo M. K., and Thomas R. 2016. Unlocking Mercury's geological history with detailed mapping of Rembrandt Basin: Year 2. Planetary Mappers' Meeting, 17, \#7023.

Irwin R. P. and Matsubara Y. 2013. Late-stage fluvial erosion in a changing climate on early Mars. AGU Fall Meeting, abstract id.P23F-1837.

Ivanov B. A. 2001. Mars/Moon cratering rate ratio estimates. Space Science Reviews 96:87-104.

Ivanov B. A. 2006. Earth/Moon impact rate comparison: Searching constraints for lunar secondary/primary cratering proportion. Icarus 183:504-507.

Ivanov B. A., Neukum G., Bottke W. F. Jr., and Hartmann W. K. 2002. The comparison of size-frequency distributions of impact craters and asteroids and the planetary cratering rate. In Asteroids III, edited by Bottke W. F. Jr., Cellino A., Paolicchi P., and Binzel R. P. Tucson, Arizona: The University of Arizona Press. pp. 89-101.

Johnson T. V. and Soderblom L. A. 1982. Volcanic eruptions on Io: Implications for surface evolution and mass loss. In Satellites of Jupiter, edited by Morrison D. Tucson, Arizona: The University of Arizona Press. pp. 634-646.

Kirchoff M. R. 2018. Can spatial statistics help decipher impact crater saturation? Meteoritics \& Planetary Science. https://doi.org/10.1111/maps.13014

Kirchoff M. R. and Schenk P. 2010. Impact cratering records of the mid-sized, icy saturnian satellites. Icarus 206:485-497.

Kirchoff M. R., Chapman C. R., Marchi S., Curtis K. M., Enke B., and Bottke W. F. 2013. Ages of large lunar 
impact craters and implications for bombardment during the Moon's middle age. Icarus 225:325-341.

König B. 1977. Investigations of primary and secondary impact structures on the moon and laboratory experiments to study the ejecta of secondary particles. Translation of Untersuch. von Primaren und Sekundaeren Einschlagstrukturen auf dem Mond und Labexperimente zum Studium des Auswurfs von Sekundaerteilchen, Ph.D. dissertation, Ruprecht Karl University, Heidelberg, Baden-Württemberg, Germany.

Krishna N. and Kumar P. S. 2016. Impact spallation processes on the Moon: A case study from the size and shape analysis of ejecta boulders and secondary craters of Censorinus crater. Icarus 264:274-299.

Landis M. E., Byrne S., Daubar I. J., Herkenhoff K. E., and Dundas C. M. 2016. A revised surface age for the north polar layered deposits of Mars. Geophysical Research Letters 43:3060-3068.

Le Feuvre M. and Wieczorek M. A. 2011. Nonuniform cratering of the Moon and a revised crater chronology of the inner solar system. Icarus 214:1-20.

Levison H. F. and Duncan M. J. 1994. The long-term dynamical behavior of short-period comets. Icarus 108:18-36.

Lissauer J. J., Squyres S. W., and Hartmann W. K. 1988. Bombardment history of the Saturn system. Journal of Geophysical Research (Planets) 93:13,776-13,804.

Malin M. C., Edgett K. S., Posiolova L. V., McColley S. M., and Zoe Dobrea E. Z. 2006. Present-day impact cratering rate and contemporary gully activity on Mars. Science 314:1573-1577.

Marchi S., Mottola S., Cremonese G., Massironi M., and Martellato E. 2009. A new chronology for the Moon and Mercury. The Astronomical Journal 137:4936-4948.

Mazrouei S., Ghent R. R., and Bottke W. F. 2015. Has the lunar impact flux rate changed in the past billion years? (abstract \#2331). 46th Lunar and Planetary Science Conference. CD-ROM.

McEwen A. S. and Bierhaus E. B. 2006. The importance of secondary cratering to age constraints on planetary surfaces. Annual Review of Earth and Planetary Sciences 34:540-567.

McEwen A. S., Moore J. M., and Shoemaker E. M. 1997. The Phanerozoic impact cratering rate: Evidence from the farside of the Moon. Journal of Geophysical Research 102:9231-9242.

McEwen A. S., Preblich B. S., Turtle E. P., Artemieva N. A., Golombek M. P., Hurst M., Kirk R. L., Burr D. M., and Christensen P. R. 2005. The rayed crater Zunil and interpretations of small impact craters on Mars. Icarus 176:351-381.

McEwen A. S., Robbins S. J., and Bierhaus E. B. 2017a. Why are there many more large secondary craters on Mercury than on the Moon or Mars? (abstract \#2028). 48th Lunar and Planetary Science Conference. CD-ROM.

McEwen A. S., Daubar I. J., Speyerer E. J., and Robinson M. S. 2017b. New lunar impact splotches: Produced by meteoroid streams and secondary impacts (abstract \#2230). 48th Lunar and Planetary Science Conference. CD-ROM.

Melosh H. J. 1984. Impact ejection, spallation, and the origin of meteorites. Icarus 59:234-260.

Melosh H. J. 1989. Impact cratering: A geologic perspective. New York: Oxford University Press.

Michael G. G. 2013. Planetary surface dating from crater sizefrequency distribution measurements: Multiple resurfacing episodes and differential isochron fitting. Icarus 226:885890.

Moore J. M., McKinnon W. B., Spencer J. R., Howard A. D., Schenk P. M., Beyer R. A., Nimmo F., Singer K. N., Umurhan O. M., White O. L., Stern S. A., Ennico K., Olkin C. B., Weaver H. A., Young L. A., Binzel R. P., Buie M. W., Buratti B. J., Cheng A. F., Cruikshank D. P., Grundy W. M., Linscott I. R., Reitsema H. J., Reuter D. C., Showalter M. R., Bray V. J., Chavez C. L., Howett C. J. A., Lauer T. R., Lisse C. M., Parker A. H., Porter S. B., Robbins S. J., Runyon K., Stryk T., Throop H. B., Tsang C. C. C., Verbiscer A. J., Zangari A. M., Chaikin A. L., Wilhelms D. E., and New Horizons Science Team. 2016. The geology of Pluto and Charon through the eyes of New Horizons. Science 351:1284-1293.

Namiki N. and Honda C. 2003. Testing hypotheses for the origin of steep slope of lunar size-frequency distribution for small craters Earth. Planets and Space 55:39-51.

Neesemann A., Kneissl T., Schmedemann N., Walter S., Raymond C., and Russell C. T. 2014. Size-frequency distributions of small impact craters on VestaImplications for secondary cratering (abstract \#1712). 45th Lunar and Planetary Science Conference. CD-ROM.

Neukum G. 1983. Meteoritenbombardement and Datierung planetarer Oberflächen. Habilitation dissertation for faculty membership. University of Munich, Munich, Germany. 186 p.

Neukum G. and Ivanov B. A. 1994. Crater size distributions and impact probabilities on Earth from lunar, terrestrialtype planet, and asteroid cratering data. In Hazards due to comets and asteroids, edited by Gehrels T. Tucson, Arizona: The University of Arizona Press. pp. 359-416.

Neukum G., Koenig B., and Arkani-Hamed J. 1975. A study of lunar impact crater size-distributions. The Moon 12:201-229.

Neukum G., Ivanov B. A., and Hartmann W. K. 2001. Cratering records in the inner solar system in relation to the lunar reference system. Space Science Reviews 96:55-86.

Ostrach R. O., Robinson M. S., Whitten J. L., Fassett C. I., Strom R. G., Head J. W., and Solomon S. C. 2015. Extent, age, and resurfacing history of the northern smooth plains on Mercury from MESSENGER observations. Icarus 250:602-622.

Plescia J. B. 2012. Uncertainties in the $<3$ Ga lunar impact cratering chronology (abstract \#1614). 43rd Lunar and Planetary Science Conference. CD-ROM.

Plescia J. B. and Cintala M. J. 2012. Impact melt in small lunar highland craters. Journal of Geophysical Research 117:E00H12.

Plescia J. B. and Robinson M. S. 2015. Lunar self-secondary cratering: Implications for cratering and chronology (abstract \#2535). 46th Lunar and Planetary Science Conference. CD-ROM.

Plescia J. B., Robinson M. S., and Paige D. A. 2010. Giordano Bruno: The young and the restless (abstract \#2038). 41st Lunar and Planetary Science Conference. CDROM.

Preblich B. S., McEwen A. S., and Studer D. M. 2007. Mapping rays and secondary craters from the Martian crater Zunil. Journal of Geophysical Research 112(E5). https://doi.org/10.1029/2006JE002817.

Quantin C., Mangold N., Hartmann W. K., and Allemand P. 2007. Possible long-term decline in impact rates. 1. Martian geological data. Icarus 186:1-10. 
Quantin C., Popova O., Hartmann W. K., and Werner S. C. 2016. Young Martian crater Gratteri and its secondary craters. Journal of Geophysical Research: Planets 121:11181140.

Ramesh K. T., Hogan J. D., Kimberley J., and Stickle A. 2015. A review of mechanisms and models for dynamic failure, strength, and fragmentation. Planetary and Space Science 107:10-23.

Robbins S. J. 2014. New crater calibrations for the lunar crater-age chronology. Earth and Planetary Science Letters 403:188-198.

Robbins S. J. 2016. Developing a global lunar crater database, complete for craters $\geq 1 \mathrm{~km}$ (abstract \#1525). 47th Lunar and Planetary Science Conference. CD-ROM.

Robbins S. J. and Hynek B. M. 2011a. Distant secondary craters from Lyot crater, Mars, and implications for surface ages of planetary bodies. Geophysical Research Letters 38. https://doi.org/10.1029/2010GL046450.

Robbins S. J. and Hynek B. M. 2011b. Secondary crater fields from 24 large primary craters on Mars: Insights into nearby secondary crater production. Journal of Geophysical Research 116(E10). https://doi.org/10.1029/ 2011 je003820.

Robbins S. J. and Hynek B. M. 2012. A new global database of Mars impact craters $\geq 1 \mathrm{~km}$ : 1. Database creation, properties, and parameters. Journal of Geophysical Research 117(E5). https://doi.org/10.1029/2011je003967v.

Robbins S. J. and Hynek B. M. 2014. The secondary crater population of Mars. Earth and Planetary Science Letters 400:66-76.

Robbins S. J., Hynek B. M., Lillis R. J., and Bottke W. F. 2013. Large impact crater histories of Mars: The effect of different model crater age techniques. Icarus 225:173184.

Robbins S. J., Bierhaus E. B., and Dones L. H. 2015. Craters of the Saturnian Satellite System: II. Mimas and Rhea (abstract \#1654). 46th Lunar and Planetary Science Conference. CD-ROM.

Robbins S. J., Singer K. N., Bray V. J., Schenk P., Lauer T. R., Weaver H. A., Runyon K., McKinnon W. B., Beyer R. A., Porter S., White O. L., Hofgartner J. D., Zangari A. M., Moore J. M., Young L. A., Spencer J. R., Binzel R. P., Buie M. W., Buratti B. J., Cheng A. F., Grundy W. M., Linscott I. R., Reitsema H. J., Reuter D. C., Showalter M. R., Tyler G. L., Olkin C. B., Ennico K. S., Stern S. A., and the New Horizons LORRI, MVIC Instrument Teams. 2017. Craters of the Pluto-Charon system. Icarus 287:187-206.

Roberts W. A. 1964. Secondary craters. Icarus 3:348-364.

Robinson M. S., Boyd A. K., Denevi B. W., Lawrence S. J., McEwen A. S., Moser D. E., Povilaitis R. Z., Stelling R. W., Suggs R. M., Thompson S. D., and Wagner R. V. 2015. New crater on the Moon and a swarm of secondaries. Icarus 252:229-235.

Rodriguez J. A. P., Gulick V. C., Baker V. R., Platz T., Fairen A. G., Miyamoto H., Kargel J. S., Rice J. W., and Glines N. 2014. Evidence for Middle Amazonian catastrophic flooding and glaciation on Mars. Icarus 2014:202-210.

Schultz P. H. and Gault D. E. 1985. Clustered impacts: Experiments and implications. Journal of Geophysical Research 90:3701-3732.

Shoemaker E. M. 1962. Interpretation of lunar craters. In Physics and astronomy of the Moon, edited by Kopal Z. London: Academic Press. pp. 283-359.
Shoemaker E. M. 1965. Preliminary analysis of the fine structure of the lunar surface in Mare Cognitum. In Ranger 7, Part 2, Experimenters' Analyses and Interpretations. JPL/NASA Technical Report 32-700, pp. $75-134$.

Shoemaker E. M., Batson R. M., Holt H. E., Morris E. C., Rennilson J. J., and Whitaker E. A. 1968. III. Television observations from Surveyor VII. In Surveyor 7 mission report. Part 2-Science results, JPL Technical Report 321264, pp. 9-76, JPL.

Shoemaker E. M., Robinson M. S., and Eliason E. M. 1994. The south pole region of the Moon as seen by Clementine. Science 266:1851-1854.

Singer K. N., McKinnon W. B., and Nowicki L. T. 2013. Secondary craters from large impacts on Europa and Ganymede: Ejecta size-velocity distributions on icy worlds, and the scaling of ejected blocks. Icarus 226:865884.

Singer K. N., Jolliff B. L., and McKinnon W. B. 2014. Lunar secondary craters measured using LROC imagery: Size-velocity distributions of ejected fragments (abstract \#1162). 45th Lunar and Planetary Science Conference. CD-ROM.

Singer K. N., McKinnon W. B., Jolliff B. L., and Plescia J. B. 2015. Icy satellite and lunar ejecta from mapping of secondary craters: Implications for sesquinary forming fragments. Workshop on Issues in Crater Studies and the Dating of Planetary Surfaces, held 19-22 May 2015, Laurel, Maryland. LPI Contribution 1841. Houston, Texas: Lunar and Planetary Institute. 9034 p.

Singer K. N., McKinnon W. B., Robbins S. J., Schenk P. M., Greenstreet S., Gladman B., Parker A. H., Stern S. A., Bray V. J., Weaver H. A., Beyer R. A., Young L. A., Spencer J. R., Moore J. M., Olkin C. B., Ennico K., Binzel R. P., and Grundy W. M., New Horizons Geology, Geophysics Team, New Horizons Composition Team, New Horizons Mvic Team, New Horizons Lorri Team. 2016. Craters on Pluto and Charon-Surface ages and impactor populations (abstract \#2310). 47th Lunar and Planetary Science Conference. CD-ROM.

Soderblom L. A., Condit C. D., West R. A., Herman B. M., and Kreidler T. J. 1974. Martian planetwide crater distributions-Implications for geologic history and surface processes. Icarus 22:239-263.

Speyerer E. J., Povilaitis R. Z., Robinson M. S., Thomas P. C., and Wagner R. V. 2016. Quantifying crater production and regolith overturn on the Moon with temporal imaging. Nature 538:215-218.

Strom R. G. 1977. Origin and relative age of lunar and Mercurian intercrater plains. Physics of the Earth and Planetary Interiors 15:156-172.

Strom R. G., Chapman C. R., Merline W. J., Solomon S. C., and Head J. W. 2008. Mercury cratering record viewed from MESSENGER's first flyby. Science 321:79-81.

Strom R. G., Banks M. E., Chapman C. R., Fassett C. I., Forde J. A., Head J. W. III, Merline W. J., Prockter L. M., and Solomon S. C. 2011. Mercury crater statistics from MESSENGER flybys: Implications for stratigraphy and resurfacing history. Planetary and Space Science 59:1960-1967.

Strom R. G., Malhotra R., Xiao Z.-Y., Takashi I., Fumi Y., and Ostrach L. R. 2015. The inner solar system cratering record and the evolution of impactor populations. Research in Astronomy and Astrophysics 15:407. 
Tornabene L. L., Moersch J. E., McSween H. Y., McEwen A. S., Piatek J. L., Milam K. A., and Christensen P. R. 2006. Identification of large $(2-10 \mathrm{~km})$ rayed craters on Mars in THEMIS thermal infrared images: Implications for possible Martian meteorite source regions. Journal of Geophysical Research 111(E10). https://doi.org/10.1029/2005je002600.

Tricarico P. 2016. The near-Earth asteroid population from two decades of observations. Icarus 284:416-423.

Van der Bogert C. H., Hiesinger H., Dundas C. M., Krüger T., McEwen A. S., Zanetti M., and Robinson M. S. 2017. Origin of discrepancies between crater size-frequency distributions of coeval lunar geologic units via target property contrasts. Icarus 298:49-63.

Vickery A. M. 1986. Size-velocity distribution of large ejecta fragments. Icarus 67:224-236.

Vickery A. M. 1987. Variation in ejecta size with ejection velocity. Geophysical Research Letters 14:726-729.

Wagner R. V., Nelson D. M., Plescia J. B., Robinson M. S., Speyerer E. J., and Mazarico E. 2017. Coordinates of anthropogenic features on the Moon. Icarus 283:92-103.

Wells K. S., Campbell D. B., Campbell B. A., and Carter L. M. 2010. Detection of small lunar secondary craters in circular polarization ratio radar images. Journal of Geophysical Research (Planets) 115(E6). https://doi.org/10. 1029/2009je003491.

Werner S. C. 2008. The early Martian evolution-Constraints from basin formation ages. Icarus 195:45-60.

Werner S. C. and Tanaka K. L. 2011. Redefinition of the crater-density and absolute-age boundaries for the chronostratigraphic system on Mars. Icarus 215:603-607.

Werner S. C., Ivanov B. A., and Neukum G. 2009. Theoretical analysis of secondary cratering on Mars and an image-based study on the Cerberus Plains. Icarus 200:406-417.

Wilhelms D. E., Oberbeck V. R., and Aggarwal H. R. 1978. Size-frequency distributions of primary and secondary lunar impact craters. Proceedings, 9th Lunar and Planetary Science Conference. pp. 3735-3762.

Williams J.-P., Paige D. A., Plescia J. B., Pathare A. V., and Robinson M. S. 2014a. Crater size-frequency distributions on the ejecta of Giordano Bruno (abstract \#2882). 45th Lunar and Planetary Science Conference. CD-ROM.
Williams J.-P., Pathare A. V., and Aharonson O. 2014b. The production of small primary craters on Mars and the Moon. Icarus 235:23-36.

Williams J.-P., van der Bogert C. H., Pathare A. V., Michael G. G., Kirchoff M. R., and Hiesinger H. 2018. Can we date very young terrestrial planetary surfaces? Meteoritics \& Planetary Science. https://doi.org/10.1111/ maps. 12924

Wisdom J. and Holman M. 1991. Symplectic maps for the nbody problem. The Astronomical Journal 102:1528-1538.

Xiao Z. 2016. Size-frequency distribution of different secondary crater populations: 1. Equilibrium caused by secondary impacts. Journal of Geophysical Research: Planets 121:2404-2425.

Xiao Z. and Strom R. G. 2012. Problems determining relative and absolute ages using the small crater population. Icarus 220:254-267.

Xiao Z., Prieur N. C., and Werner S. C. 2016. The selfsecondary crater population of the Hokusai crater on Mercury. Geophysical Research Letters 43:7424-7432.

Zahnle K., Schenk P., Levison H. F., and Dones L. 2003. Cratering rates in the outer solar system. Icarus 163:263289.

Zahnle K. J., Alvarellos J. L., Dobrovolskis A. R., and Hamill P. 2008. Secondary and sesquinary craters on Europa. Icarus 194:660-674.

Zanetti M., Stadermann A., Krüger T., van der Bogert C., Hiesinger H., and Jolliff B. L. 2014. Mapping crater density variation on Copernican ejecta blankets: Evidence for auto-secondary cratering at Tycho and Aristarchus (abstract \#1528). 45th Lunar and Planetary Science Conference. CD-ROM.

Zanetti M., Stadermann A. C., Jolliff B. L., Hiesinger H., and van der Bogert C. H. 2015. Making the case for autosecondary cratering: Evidence from young lunar ejecta blankets. Issues in Crater Studies and the Dating of Planetary Surfaces 1841:9041.

Zanetti M., Jolliff B., van der Bogert C. H., Hiesinger H., Plescia J., and Artemieva N. 2016. Self-secondary crater populations on Copernican continuous ejecta blankets. LPI Contribution 1911. Houston, Texas: Lunar and Planetary Institute. 6019 p. 